\title{
The dynamic stage of clusters and its influence on the stellar populations of galaxies
}

\author{
N. R. Soares, ${ }^{1 \star}$ S. B. Rembold ${ }^{1}$ \\ ${ }^{1}$ Universidade Federal de Santa Maria - 97105-900, Santa Maria-RS, Brazil
}

Accepted XXX. Received YYY; in original form ZZZ

\begin{abstract}
We investigate the stellar populations of galaxies in clusters at different dynamical stages, aiming to identify possible effects of the relaxation state of the cluster or subcluster on the star formation histories of its galaxies. We have developed and applied a code for kinematic substructure detection to a sample of 412 galaxy clusters drawn from the Tempel et al. (2012) catalogue, finding a frequency of substructures of $45 \%$. We have extracted mean stellar ages with the STARLIGHT spectral synthesis code applied to SDSS-III spectra of the sample galaxies. We found lower mean stellar ages in unrelaxed clusters relative to relaxed clusters. For unrelaxed clusters, we separated primary and secondary subhalos and found that, while relaxed clusters and primaries present similar masses and age distributions, secondaries present younger stellar populations, mainly due to low-mass galaxies $\left(\log M_{\star} / M_{\odot} \lesssim 11 \mathrm{dex}\right)$. An age-clustercentric radius relation is seen for all subhalos irrespective of the presence of substructures. We also observe relations between the mean stellar age and mass of relaxed and unrelaxed clusters, massive systems presenting higher mean ages. The locus of these relations is distinct between relaxed and unrelaxed clusters, but become indistinguishable when separating primaries and secondaries. Our results suggest that differences between relaxed and unrelaxed clusters are mainly driven by low-mass systems in the clusters outskirts, and that, while pre-processing can be seen in the subcomponents of dynamically young clusters, some evolution in the stellar populations must occur during the clusters relaxation.
\end{abstract}

Key words: galaxies: clusters: general; galaxies: evolution

\section{INTRODUCTION}

In the current scenario of hierarchical formation, galaxy clusters are formed through a series of mergers of smaller systems and are the most massive structures that have decoupled from the expansion of the universe (Press \& Schechter 1974; Jõeveer et al. 1978; van den Bergh 1999; Kauffmann et al. 1999). In this scenario, clusters of galaxies grow by accretion of both field galaxies and bounded structures like low-mass groups. The process of accretion of lower mass systems by a cluster - or even the build up of a single massive cluster from merging of two individual clusters - is known to imprint distinct signatures in the cluster properties before full virialization of the system. These signatures, in the form of asymmetric, extended X-ray distributions (Kapferer et al. 2006; Ferrari et al. 2006; Parekh et al. 2015), multiple peaks in the galaxy density field (e.g. Boschin, Barrena

^ E-mail: ruschelsoares@gmail.com.br
\& Girardi 2009; Barrena et al. 2009), or distinctive line-ofsight galaxy velocity distributions (e.g. Ribeiro et al. 2010; Barrena et al. 2014), are usually referred to as "substructures". Clusters and groups can be dynamically "relaxed" or "unrelaxed" according to the presence or absence of substructures. Besides being an important tool for evaluating models of formation and evolution of large scale structures in the Universe (Natarajan \& Volker 2004; Gao et al. 2004; van den Bosch \& Jiang 2016; Schwinn et al. 2017; Natarajan et al. 2017), the evolutionary stage of a galaxy cluster is important in understanding the evolution of the baryons (gas and stars) in the galaxies themselves.

It is well known that in the central regions of clusters of galaxies the most common morphology of galaxies found are early-type (Hogg et al. 2003; Zhu et al. 2010; Jaffé et al. 2016; Deshev et al. 2017; Sybilska et al. 2017) and dominated by older stellar populations (Bower et al. 1999) when comparing to field galaxies, which are mostly late-types with a large 
contribution of younger stellar populations (Dressler 1980; Kelkar et al. 2017; Oh et al. 2018).

This dichotomy can arise both by the initial conditions in which galaxies were first formed and/or by the later transformation of the galaxy morphology and stellar population properties due to the environment. While recent works demonstrate that at least part of the presenttime galaxy properties are set early in the cosmic history, favouring the so-called "nature" scenario (e.g. Athanassoula 2010; Shi et al. 2017), there is mounting evidence suggesting that the environment is important in the transformation of the galaxy stellar content (Poudel et al. 2017; Hwang et al. 2018; Crone Odekon et al. 2018). All in all, high density regions are associated to the quenching of the star formation in gas-rich galaxies and subsequent morphological transformations associated to the loss of a gaseous disk. This quenching process may not be limited to the highdensity cluster environment, as a large body of observational evidence have shown that infalling galaxies in the clusters outskirts present properties that are markedly distinct from those of field galaxies, what suggests that such late arrivals have already been pre-processed (Wetzel et al. 2013; Hou et al. 2014). An extensive zoology of environmentallydriven evolutionary processes have been proposed theoretically to account for the star formation quenching in highdensity environments and pre-processing signatures, and have been confronted with the observations with variable degrees of success. These processes include ram-pressure stripping, tidal stripping, strangulation and galaxy harassment (Fujita \& Nagashima 1999; Ruggiero \& Lima Neto 2017; Balogh, Navarro \& Morris 2000; Peng, Maiolino \& Cochrane 2015; Park \& Hwang 2009; Hernández-Fernández, Vílchez \& Iglesias-Páramo 2012). These effects are thought to present different sensitivities to the environment and occur in different timescales, so a combination of them can be necessary to produce the observed environmental trends (e.g. Bahé et al. 2013; Cora et al. 2018).

The environmental density at the location of a given galaxy does not seem, however, to be the only relevant parameter shaping its stellar population properties. It has been found that galaxy properties are distinct between relaxed and unrelaxed clusters, though the details are still unclear. Ribeiro, Lopes \& Rembold (2013) have shown, for a large sample of low- and high-mass clusters, that relaxed clusters, characterized by a Gaussian velocity distribution, present a higher fraction of red faint galaxies than unrelaxed clusters, while a color-radius relation was detected for both relaxed and unrelaxed structures. In Ribeiro et al. (2013), on the other hand, no significant radial segregation of galaxy properties have been found for unrelaxed clusters, and most of the differences in the stellar population properties between relaxed and unrelaxed clusters was found to occur for lowmass galaxies; this last result was also found by Carollo et al. (2013), but the intensity of the detected signal was weak. Cohen et al. (2014) and Cohen, Hickox \& Wegner (2015) have found a higher star formation rate in galaxies residing in unrelaxed clusters. de Carvalho (2017) have found a larger fraction of old, high-metallicity faint galaxies in the outskirts of unrelaxed clusters as compared to relaxed ones, what was interpreted as due to infall of galaxies pre-processed in groups. Roberts \& Parker (2017), on the other hand, have found no significant difference in the stellar populations of galaxies outside the virial radius of relaxed and unrelaxed clusters, but at smaller clustercentric distances unrelaxed clusters present a higher star formation rate than relaxed clusters. Hou et al. (2012) have found an increase in the blue population and star forming galaxies in clusters where kinematic substructures, detected by means of the DresslerSchectman test (Dressler \& Shectman 1988), are present. Guennou et al. (2014), identifying substructures in clusters by means of X-ray imaging, have found that substructures which are located near the cluster centers are depleted in late-type galaxies with recent bursts of star formation.

Most of these works do not directly address if the infall or cluster merging processes are helping to shape the star formation history of the galaxies. A number of works investigating binary pairs of merging clusters (e.g. Ferrari et al. 2005; Hwang \& Lee 2009; Stroe et al. 2015; Wegner, Chu \& Hwang 2015) have found evidences which suggest an enhancement of star formation in galaxies. These results are often interpreted as the triggering of star formation in an infalling gas-rich galaxy due to shocks in the intracluster medium (ICM) or ram pressure (Bekki, Owers \& Couch 2010; Ebeling et al. 2014; Roediger et al. 2014), though other works suggest that the high merger-driven ICM densities result in quenching of star formation (e.g. Fujita et al. 1999; Boselli \& Gavazzi 2006). Mansheim et al. (2017) have found some hints of recent star formation episodes that could be related to the merging process occurring in DLSCL J0916.2+2953, but this evidence is inconclusive. Ma et al. (2010) have found evidence for a merging-induced starburst following the pericentric passage of MACSJ0025.4-1225, resulting in a distribution of $\mathrm{E}+\mathrm{A}$ galaxies along the merging axis. However, Deshev et al. (2017) have found evidence for quenching along the merger axis of the merging cluster A520 but no sign of merging-induced starbursts in the gas-rich galaxy population. A similar result was obtained by Pranger et al. (2013) and Pranger et al. (2014) for the merging clusters Abell and 3921 Abell 2384 respectively. It is, therefore, still open to debate the impact of the merging process itself in unrelaxed clusters on the stellar population properties, and how much of the observed differences between the stellar population properties of galaxies in relaxed and unrelaxed clusters can be attributed solely to the late arrival of infalling halos. In particular, how are the stellar population in substructures compared to relaxed clusters of similar mass? Do individual substructures in unrelaxed clusters present evidence of pre-processing? What is the relative impact in relaxed and unrelaxed clusters on the SFH of galaxies with different stellar masses? Does the pre-processing occur in all halo mass scales?

In this work, we investigate differences between stellar populations in galaxies in clusters with different dynamic stages, aiming to identify the influence of environmental effects in the galaxy stellar populations by the decomposition of unrelaxed clusters in their constituent subhalos, trying to shed some light on the above questions. For this purpose, we use a sample of clusters of groups and clusters selected from the Tempel et al. (2012) catalogue, based on the eighth data release of SDSS III. To identify substructures and characterize their kinematic properties, masses and radii, we developed an automated algorithm based on the $k$-test of Colles \& Dunn (1996). We use the STARlight CODE (Cid et al. 
2005; Mateus et al. 2006) to perform the stellar population synthesis and derive the mean stellar age of galaxies.

The paper is organized as follows: in Section 2 we describe our data and catalogue; in Section 3 we describe our methodology for substructure identification and characterization, and also the derivation of the stellar population properties of the galaxies in these structures. In Section 4 we present the results, where we investigate how the stellar populations of galaxies depend on the properties of the structure they reside in, and in Section 5 we discuss and summarize our conclusions. In this work we assumed a cosmology with $\Omega_{m}=0.3, \Omega_{\Lambda}=0.7$ and $\mathrm{H}_{0}=70 \mathrm{kms}^{-1} \mathrm{Mpc}^{-1}$

\section{DATA}

\subsection{Cluster and group sample}

In this work, we intend to analyze the stellar populations of galaxies in groups and clusters spanning the largest range possible in richness and dynamic stage. For our purposes, it is convenient to rely on a large, homogeneous catalogue, with a strict identification of confirmed members and interlopers (i.e. free from projection effects), and for which optical spectra are available. The Tempel et al. (2012) catalogue (hereafter TTL) is a large spectrophotometric catalogue implemented on the eighth data release of Sloan Digital Sky Survey (Aihara et al. 2011). This catalogue contains 77,858 groups with more than three confirmed members and spanning the range $0.009<z<0.2$. The groups are identified in the redshift-projected position space with the friends-offriends algorithm with a variable linking length to avoid selection effects. As we describe in Sect. 3, the characterization of the dynamical stage of a cluster using galaxy velocities relies on a large number of spectroscopically confirmed members. We have thus selected from this catalogue all clusters with more than 30 galaxies, what resulted in a sample of 412 clusters, comprising 24,169 galaxies. From this preliminary list, we excluded eight objects (identified by the codes 09349, 13462, 20575, 24918, 33262, 34727, 49298 and 62138) which correspond to superclusters and filaments and are too complex for our analysis.

Using the CASJOBS server $^{1}$, we have obtained the SDSS-III optical spectra for all galaxies in our sample from Data Release 10 (Ahn et al. 2014). These single-fiber spectra cover the wavelength region $3800-9200 \AA$ with a spectral resolution $\sim 2000$ and cover the inner 3 arcseconds of the galaxy. We have also obtained the absolute magnitudes in the $r$ band as derived from the SDSS photometric redshift pipeline. Because some galaxies lack an estimate of photometric redshift, the final sample of cluster galaxies was slightly reduced to 20,192 galaxies.

\section{$2.2 \quad$ Field sample}

In order to better constrain the impact of the cluster evolutionary stage on member galaxies, it is important to compare the general properties of galaxies in clusters with field galaxies. We have therefore selected a control sample of field galaxies - i.e. galaxies not associated to clusters or

1 http://skyserver.sdss.org/casjobs/ groups - which are otherwise similar to out sample of cluster galaxies in stellar mass and redshift. Using the CASJOBS server, we have selected a preliminary field sample with 200,000 galaxies with the same $r$-band apparent magnitude as the TTL sample and selected from SDSS-III DR-10. We then measured the maximum projected clustercentric distance $D_{\max }$ and the maximum peculiar velocity $V_{\max }$ of all galaxies in the TTL sample with relation to its parent cluster/group. A galaxy from our preliminary list was then confirmed as a field galaxy if (a) its projected distance to each cluster center exceeds $2 \times D_{\max }$ or (b) its line-of-sight velocity offset relative to all clusters in the TTL sample exceeds $V_{\max }+2000 \mathrm{~km} \mathrm{~s}^{-1}$. After performing these cuts, the field sample was reduced to 35,402 galaxies. For this preliminary sample, we obtained the SDSS-III single-fiber spectra and the $r$-band values of the absolute, apparent and fiber magnitude. These galaxy spectra were then used to estimate their stellar masses and mean stellar ages (see Sect. 3.3).

The next step was to compare the stellar mass and redshift of galaxies in field sample with those of galaxies in our cluster sample. For this, we introduce the metric

$\mu=\sqrt{\left(\frac{z-z_{f l d}}{0.05}\right)^{2}+\left(\frac{M_{\star}-M_{\star f l d}}{2 \times 10^{11.5}}\right)^{2}}$,

where $z$ and $M_{\star}$ are the redshift and the stellar mass of galaxies in the cluster sample (sect. 3.3), and $z_{f l d}$ and $M_{\star f l d}$ are these same parameters for field galaxies. For every galaxy in the cluster sample we have then selected the field galaxy which minimized $\mu$. The final field sample comprises therefore the same number of galaxies as our sample of cluster galaxies. In Figure 1 we compare the stellar mass and redshift distributions of galaxies in the field and cluster samples.

\section{SUBSTRUCTURE DETECTION}

Our main objective is to analyze the differences between galaxies that reside in clusters in different dynamical stages through their stellar populations, and for this we developed a method that is able to (automatically) detect substructures in galaxy clusters and derive their kinematic parameters. The Dressler-Schectman $(\delta)$ test (Dressler \& Shectman 1988 ) and the $\kappa$-test (Colles \& Dunn 1996) are 3D tests that have been used to detect substructures in clusters. However, the identification of each substructure and of the galaxies therein usually relies on the visual analysis of "bubble plots", where galaxies are represented as circles proportional to the size of a statistical parameter which is sensitive to differences between the local and the global velocity distributions. In the $\delta$ test, for each galaxy $i$ and its $N_{n}$ nearest neighbors in the projected position space, the $\delta_{i}$ statistics for each $i$ galaxy is defined by:

$\delta_{i}^{2}=\frac{N_{n}}{\sigma_{g}^{2}}\left[\left(v_{l}-v_{g}\right)^{2}+\left(\sigma_{l}-\sigma_{g}\right)^{2}\right]$

where $v_{l}\left(v_{g}\right)$ is the local (global) mean velocity, and $\sigma_{l}\left(\sigma_{g}\right)$ is the local (global) velocity dispersion. A local "cloud" of large $\delta_{i}$ values is interpreted as evidence for the presence of a substructure. The summation of all $\delta_{i}$ values produces the $\Delta$ statistics that, when compared to the typical values obtained from Monte Carlo shuffling the velocities of the galaxies in the cluster, can be used to infer the overall evolutionary stage of the cluster. 

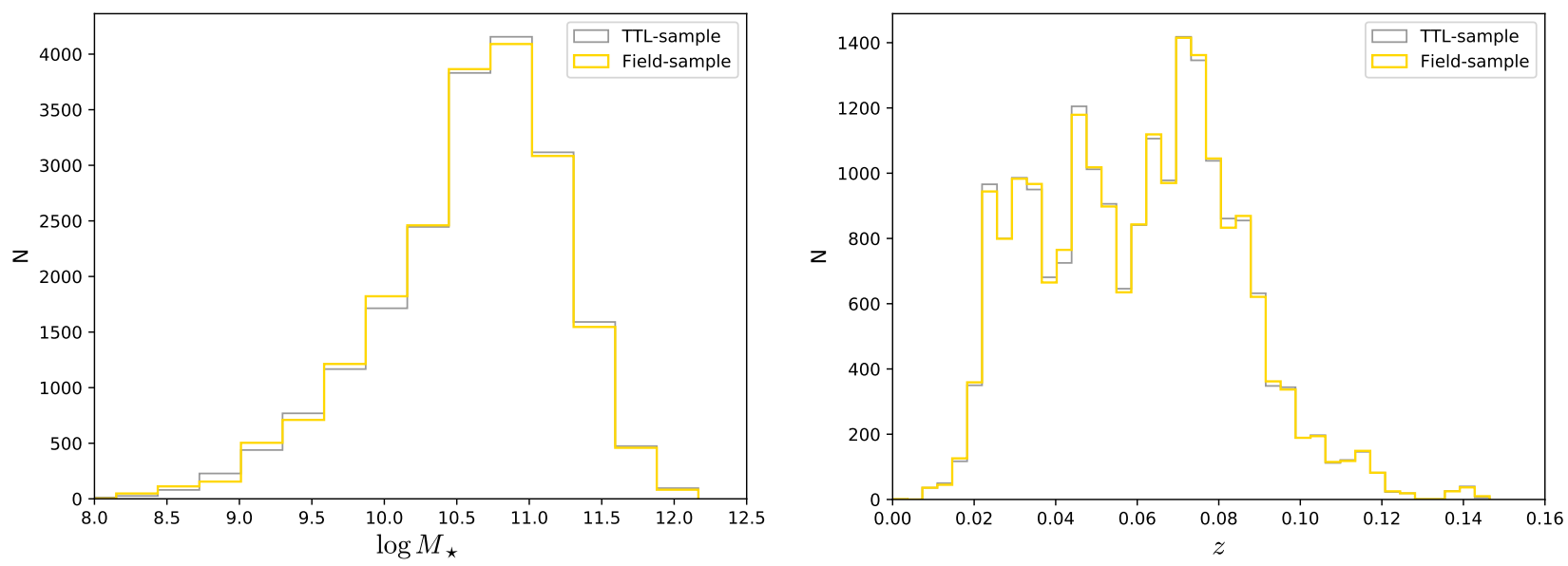

Figure 1. Stellar mass (left) and redshift (right) distributions of galaxies in our cluster (grey) and field (yellow) samples.

The $k$-test of Colles \& Dunn (1996) is similar to the $\delta$ test, but the $\kappa$ statistic is derived from the comparison of the full local/global velocity distributions, as opposed to the $\delta$ statistics and its dependence only on the first two moments of the distribution. For each $i$ galaxy, the $\kappa_{i}$ statistics calculated by:

$k_{i}=-\log P_{K S}\left(D>D_{o b s}\right)$

where $\mathrm{P}_{K S}$ is the Kolmogorov-Smirnov probability that the local velocity distribution and the global velocity distribution are derived from the same original distribution. The derived "bubble plots" and the summation of the $k_{i}$ values are interpreted as in the delta test. However, the $\kappa$ test has the severe limitation of being non-automated for identifying galaxies in substructures. We have therefore developed an automated algorithm - Local Kinematic Estimator (LOCKE) inspired on the $\kappa$-test that is capable of identify the individual structures and assign the galaxies to the proper structure, without visual inspection.

\subsection{LocKE - Local Kinematic Estimator}

The main goal of LocKE is to extract individual substructures and their member galaxies, providing estimates of their kinematic parameters, through comparison between the velocity distributions in different regions of the clusters. The success of this procedure is crucially dependent on the contrast between the local and global velocity distributions, and therefore on the definition of "local" and "global" galaxies - i.e. the choice of the number of neighbours $N_{n}$. Dressler \& Shectman (1988) used a fixed value of $N_{n}$ at 10 , while Aguerri \& Sánchez-Janssen (2010) used the square root of the total number of galaxies in the cluster. This choice is crucial for the correct detection of substructures and, as a general rule, the largest statistics in the $\kappa$ test, and the largest contrast between the substructure and the remainder of the cluster in the "bubble plots" will be obtained when $N_{n}$ is comparable to the number of galaxies in the substructure. This is illustrated in Figure 2, where we present the $\kappa$-test "bubble plot" for a synthetic bimodal cluster composed of a primary structure of 92 galaxies with $\sigma=878 \mathrm{~km} / \mathrm{s}$ and a secondary structure of 32 galaxies with $\sigma=518 \mathrm{~km} / \mathrm{s}$ and a line-of-sight velocity offset of $563 \mathrm{~km} / \mathrm{s}$. We have run the $\kappa$ test with variable $N_{n}$ values. For each run we show in the plots the summation of the $\kappa$ values and its corresponding percentile after 1000 realizations of Monte Carlo shuffling in the velocity space - i.e. the significance of substructure detection. Notice that both the $\kappa$ statistics and the significance of the test are lower, and the location and physical limits of the substructure become harder to define, for $N_{n}$ much larger or smaller than the number of galaxies in the substructure.

The above discussion does not take into account the presence of multiple substructures in a cluster. For a multimodal cluster comprised of one main structure and a number of secondary structures, the largest $\kappa$ values in general will be obtained for the substructure with the larger kinematic differences relative to the full velocity distribution, but other structures, when present, can usually also be visible at smaller or comparable $\kappa$ values. Individual substructures must therefore me identified in a case-by-case analysis which take into consideration the typical $\kappa$ values of each region and multiple concentrations of galaxies. We illustrate this in Figure 3, which presents the bubble plot of a simulated cluster made up of three individual kinematic structures. Notice that the two clouds of large and medium-sized $\kappa$ values are only identifiable as two structures by comparison of the local mean velocities, but not by the size of the $\kappa$ statistics. When we exclude the region associated to the largest $\kappa$ values, the bimodal structure of the remaining field becomes obvious.

The examples above show that, in order to automatically identify individual substructures in the field of a cluster using the $\kappa$ test, a stratified, multi-scale comparative analysis between the local and global kinematics of a cluster is mandatory. That is the rationale behind LocKE, developed in the Python language and whose steps and structure we describe in the following.

\subsubsection{Initialization}

LOCKE expects as input a list of sky coordinates and redshifts of the $N_{o}$ cluster member galaxies. At initialization, the nearest neighbours of each galaxy are obtained using 


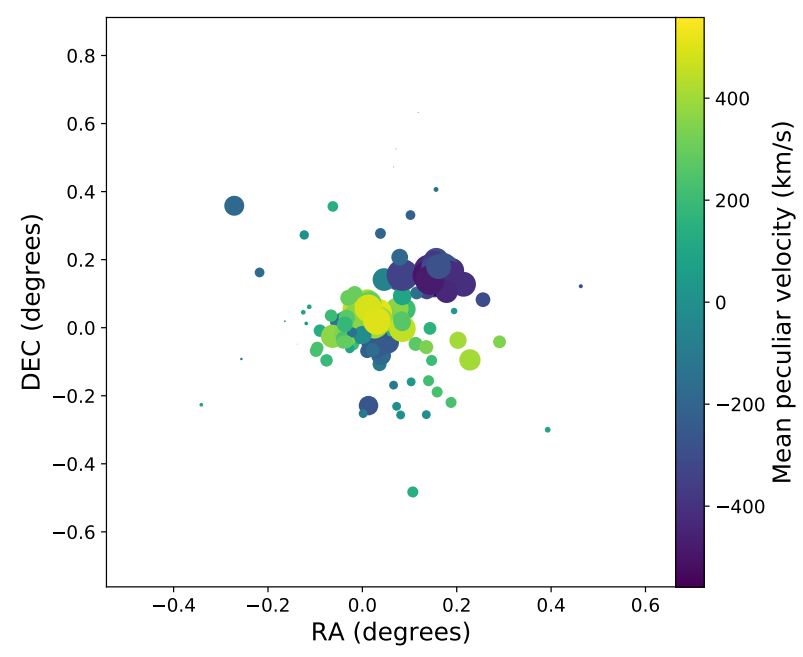

(a)

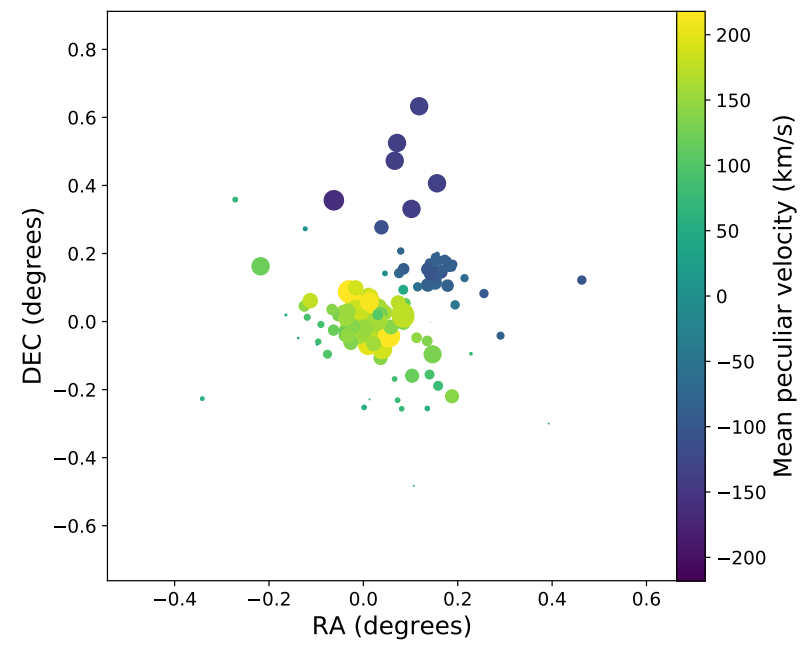

(c)

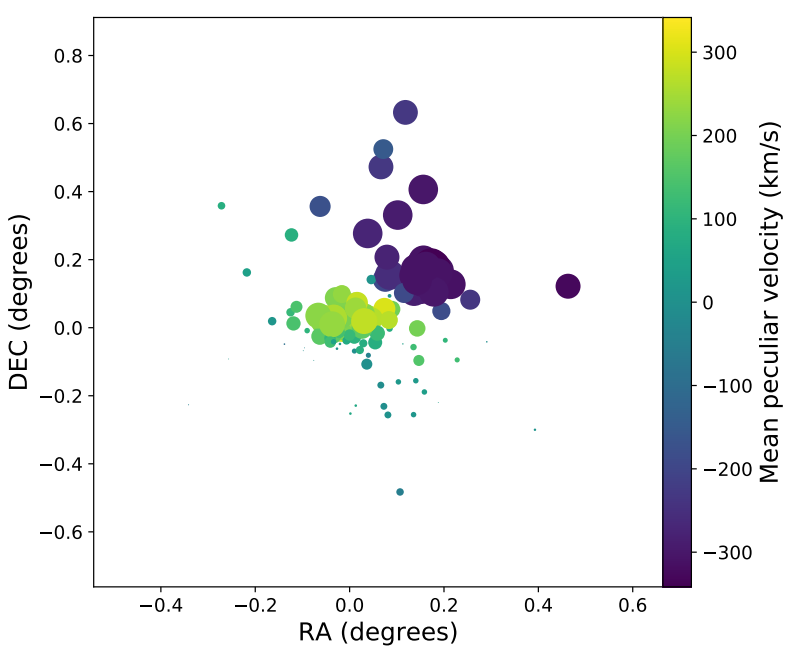

(b)

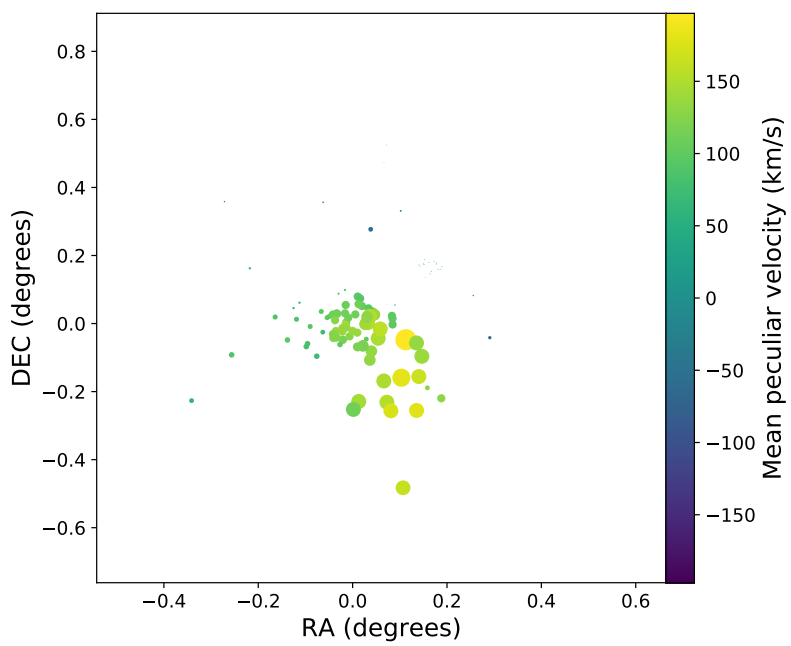

(d)

Figure 2. Bubble plots for a bimodal simulated cluster, for $N_{n}=10$ (a), 30 (b), 50 (c) and 70 (d). The size of each circle is related to the size of the $\kappa$ statistics, and the circle colours indicates the average line-of-sight velocity of the $N_{n}$ neighbours.

the BallTree algorithm implemented in the Python module scikit-learn (Pedregosa et al. 2011).

\subsubsection{Statistics maximization}

We start with a first value for the number of neighbours $N_{n}=\sqrt{N_{o}}$, which we define as the lowest possible number of neighbours which define a substructure in the $\kappa$-test bubble plots. LocKE then creates internally a grid of 10 equally-spaced values of $N_{n}$, ranging from $\sqrt{N_{o}}$ to $3 N_{o} / 4$. For each of these values, the individual $\kappa_{i}$ values are obtained for all cluster galaxies. For this calculation, we define as the "global" kinematics the velocity distribution of all galaxies except the $N_{n}$ nearest neighbours of each galaxy, instead of the full cluster velocity distribution. This is done to maximize the absolute value for the $\kappa$ statistics. LocKE then identifies the number of neighbours $N_{n, \max }$ for which the average value of $\kappa$ is maximum. The spacing between consecutive values of the $N_{n}$ grid is then reduced to one half of its initial value, and the $\kappa_{i}$ calculation is repeated, producing an updated value of $N_{n, \max }$. This procedure of reducing the spacing of the $N_{n}$ grid is repeated until convergence. The convervence value of $N_{n, \max }$ therefore corresponds to the number of neighbours which produces the best contrast between local and global velocity distribution. We will refer to the convergence value of $N_{\max }$ as $N_{n \text {,best }}$. Notice that, at this stage, we allow a "substructure candidate" to be composed of more than half of the total number of galaxies in the cluster field; we will later discuss the reason behind this choice.

\subsection{3 $\kappa$ statistics}

For the $N_{n, b e s t}$ nearest neighbours, LocKE performs a final calculation of the $\kappa_{i}$ values, as well as the summation of these values, $\kappa_{\text {clus }}$. LocKE then randomly shuffles the 


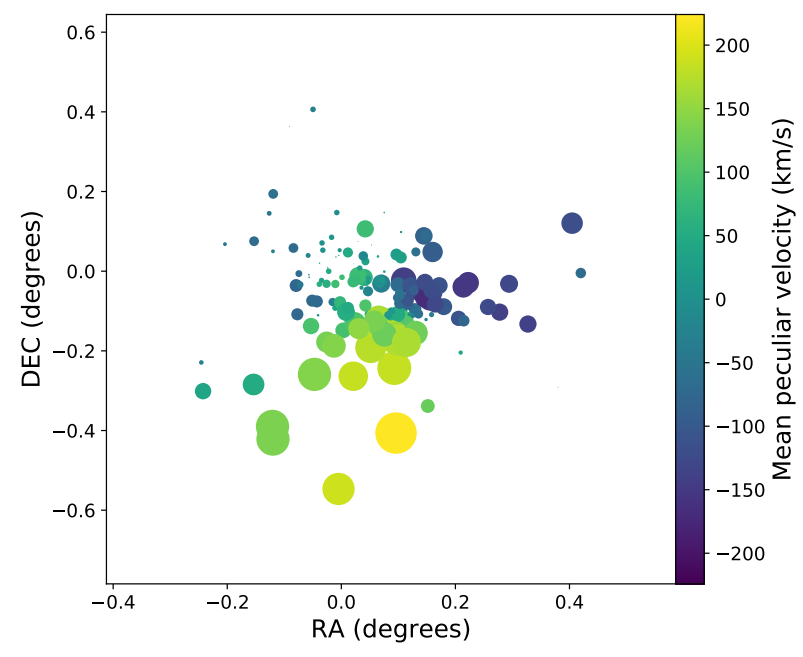

(a)

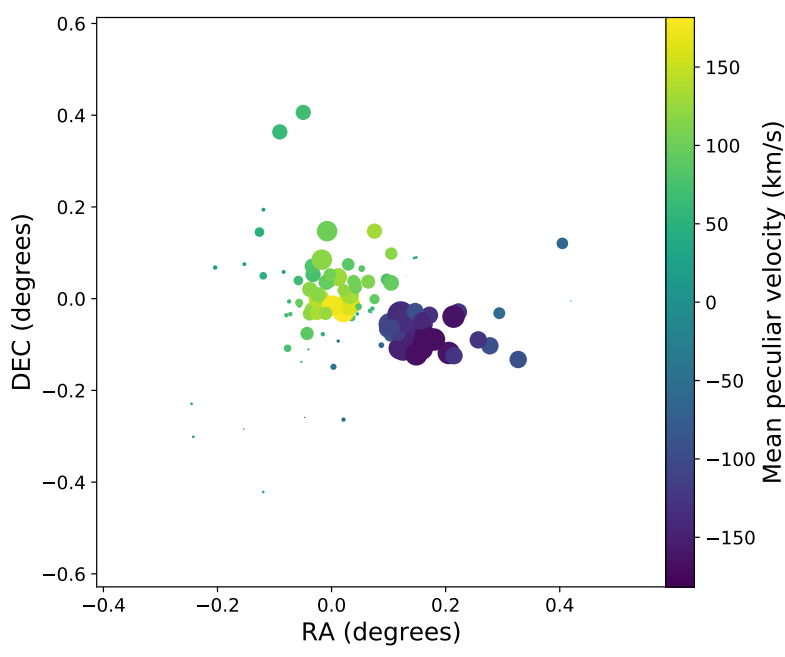

(b)

Figure 3. Bubble plots for a simulated cluster with three kinematic structures. In panel (a), all galaxies are included. In panel (b), we exclude the structure associated to the larger $\kappa$ values.

velocities of all galaxies in the cluster field and, using the same $N_{n, b e s t}$ value, calculates the respective $\kappa$ values. The significance of the observed $\kappa_{\text {clus }}$ statistics is obtained by comparison with the distribution of $\kappa$ values obtained for a large number of reshuffling runs; we consider a positive detection of substructure all cases where the significance of the test is higher than $95 \%$. In our early experiments we have found that, for a large number of clusters, the significance of the $\kappa$ test is barely higher or lower than $95 \%$, so that a very large $\left(\sim 10^{5}\right)$ number of simulations had to be performed to confirm a discard the presence of substructures in these clusters. So, instead of performing so many simulations for all clusters, even those which are clearly devoid of substructures, we follow a different approach. For $N_{s}$ blocks of 20 simulations each, we calculate the average $95 \%$ percentile $p$ of all simulated $\kappa_{i}$ values, and the standard error $\sigma$ of this percentile. We then increase $N_{s}$ until the absolute difference between $p$ and the observed $\kappa_{\text {clus }}$ value is larger than $2 \sigma-$ i.e. until $p$ itself is constrained at the $95 \%$ level. For clusters with evident substructures or lack thereof, $N_{s}$ has shown to be as low as $\sim 3-4$, resulting in a very short processing time; for other clusters, however, the $N_{s}$ value can be of the order $\sim 10^{2}$, and the processing time will be correspondingly higher. In our tests, the identification (or not) of substructures using this criterion was rigorously identical as that obtained by simply assuming a fixed large number of reshuffling operations. It is important to note that all that LocKE cares about is whether or not a substructure is detected in the field of a cluster, independently of the particular value of its statistical significance.

\subsubsection{Absence of substructures}

If the significance of substructuring is lower than 95\%, LOCKE assumes that the cluster is devoided of substructures. Using the kinematics and positions of all galaxies in the cluster, it estimates the mean line-of-sight velocity $v$ and dispersion $\sigma_{v}$, using the biweight location and scale estima- tor implemented in the astLib.astStats Python module, assuming tuning constants for location and scale of 6.0 and 9.0, respectively (Beers, Flunn \& Gebhardt 1990). This calculation is done with a $3-\sigma$ clipping algorithm which is a slight modification of the astStats.biweightClipped module. The uncertainties in both kinematical parameters are obtained with the jackknife technique (Beers, Flunn \& Gebhardt 1990).

\subsubsection{Identification of substructures}

If the significance of substructuring is higher than $95 \%$, LOCKE assumes that a potential substructure has been found. The spatial distribution of the $\kappa_{i}$ values is then used to estimate the center and the extension of the substructure detected. The map of galaxy coordinates is convolved with a Gaussian distribution, the standard deviation of which is calculated by the average distance of the $N_{n, b e s t}$ nearest neighbours of all galaxies in the cluster. At this step, the statistical weights of the convolution are the $\kappa_{i}$ values of each galaxy. This results in a continuous density map where the local density is directly associated to the size of the $\kappa$ statistics around each point. LocKE then attributes the firstguess center of the substructure to the peak of the $\kappa$ density map. The first-guess substructure member "candidates", $P$, are all the $N_{n, \text { best }}$ nearest neighbours of the galaxy closer to the density peak. If, for any of these $P$ preliminary members, the average $\kappa$ values of its $N_{n, b e s t}$ nearest neighbours is higher than for the galaxy closer to the density peak, the list of candidates $P$ is updated by replacing the galaxy closer to the density peak for the galaxy with the higher $\kappa$ value, and the substructure member candidates are replaced by its $N_{n, b e s t}$ nearest neighbours.

\subsubsection{Substructure decontamination}

The member candidates of the substructure, as identified in the previous step, usually include galaxies which are mem- 
bers of other structures in the field of the cluster. The substructure contamination will be the more severe the larger the superposition of the individual 3D structures along the line of sight. LOCKE tries to clean the substructures by means of a two-gaussian mixture modelling in the velocity space, using the mixture module. The starting parameters of gaussian mixture are the median and standard deviations of the velocity distributions of the substructure candidates and the remaining galaxies in the cluster. After convergence, galaxies are "confirmed" as substructure members if they are associated to the substructure velocity distribution by the mixture module; the remaining galaxies - usually very few, but in some cases a significant fraction of the full substructure - are considered outliers and are attributed to the remaining cluster structure. The field contamination is the reason why we allow a potential substructure to contain more than half the total number of galaxies in the field. The parameters of the velocity distribution of the substructure are assumed to be the center and width of the gaussian fit obtained by the mixture module. Uncertainties in these parameters are derived by bootstrapping the velocity distribution of the substructure candidates and running mixture module for all realizations, where the number of bootstrap operations is set by the user.

\subsubsection{Multiple substructures and dynamical parameters}

After identification of the first-level substructure, all steps are repeated in the remaining galaxy field, after excluding the confirmed substructure members. Multiple levels of substructuring can therefore be detected for the same cluster. These steps are repeated until no further substructures are detected, i.e. the significance of the $\kappa$ test is lower than $95 \%$. Having identified and extracted all individual substructures in the field of a cluster, LocKE then derives masses and physical radii for each individual structure.

The virial mass $\left(M_{v}\right)$ of the structures was measured using equation 4, assuming that the galaxy distribution in the structure follows the mass distribution and the system is linked by the same gravitational potential well (Merritt 1988; Girardi et al. 1998).

$M_{v}=\frac{3 \pi}{2} \frac{\sigma_{P}^{2} R_{P V}}{G}$,

here $\sigma_{p}$ is the projected velocity dispersion measured by LocKE and $\mathrm{R}_{P V}$ is the projected virial radius of the structure, given by

$R_{P V}=\frac{N(N-1)}{\sum_{i \neq j} R_{i j}^{-1}}$,

(Girardi et al. 1998), where $R_{i j}$ is the distance between a pair of galaxies $i$ and $j$, and $N$ is the total number of galaxies in the structure. A pressure correction term $C$ was applied on the mass estimate; the lack of this correction will overestimate the cluster masses (Carlberg et al. 1996). In this work, we measured the corrected virial mass inside a virial radius $R_{\text {vir }}$ given by

$R_{v i r}=\sqrt[3]{\frac{\sigma_{P}^{2} R_{P V}}{6 \pi H_{0}^{2}}}$, and the corrected mass is

$$
\begin{aligned}
M_{C V} & =M_{V}-C \\
& =M_{V}\left[1-4 \pi R_{v i r}^{3} \frac{\rho\left(R_{v i r}\right)}{\int_{0}^{R_{v i r}} 4 \pi r^{2} \rho d r}\left(\frac{\sigma_{r}\left(R_{v i r}\right)}{\sigma\left(<R_{v i r}\right)}\right)^{2}\right],
\end{aligned}
$$

where the parameter $\left(\sigma_{r}(b) / \sigma(<b)\right)^{2}$ is the velocity anisotropy. The anisotropy parameter is not easy to extract for a given galaxy structure, especially for very poor systems, so that instead of deriving it for each detected structure, we simply assume that all individual structures are dynamically relaxed - even though the systems they are part of can be themselves unrelaxed structures. As shown by Costa, Ribeiro \& de Carvalho (2018), relaxed clusters typically present radially decreasing velocity dispersion profiles. Velocity dispersion profiles of this type are produced by isotropic velocities in the inner region and radial velocities in the external regions, and can be well described by a velocity anisotropy parameter of 0.6 (Girardi et al. 1998). We have therefore fixed the anisotropy parameter to this value.

Finally, the $R_{200}$ radius is calculated following Yan et al. (2015),

$R_{200}=\frac{\sqrt{3} \sigma}{10 H\left(z_{c}\right)}$,

where $\sigma$ is the velocity dispersion of the structure and $H\left(z_{c}\right)$ is the Hubble factor at redshift $z_{c}$.

\subsection{LocKE performance}

A variety of methods for detecting substructures in clusters is available in the literature, but most of these do not automatically separate galaxies in the many individual cluster structures. One test that can be used for this is multidimensional normal mixture modelling. In this test, the full 3D space of the cluster galaxies is decomposed with a mixture of multidimensional Gaussians. The number of individual Gaussians found in the solution is interpreted as the amount of individual structures detected in the cluster. This multidimensional modelling, when compared to the $\delta$ or $\kappa$ tests, has the advantage that the separation of galaxies in each structure is done automatically. This method has been successfully applied by Einasto et al. (2012) to identify structures in a large sample of groups and clusters. The authors have performed the mixture modelling with the package Mclust (Fraley \& Raftery 1999). We performed some preliminary tests using simulated clusters to determine the performance of Mclust in comparison to the $\kappa$-test. In the performed tests, the $\kappa$-test performed better than Mclust for correctly identifying substructures whose centroids do not overlap spatially; this, in fact, was one of the motivations behind LocKE development. In the following, we quantify the LocKE performance taking the Mclust performance as a reference.

For this, we have created a set of 3000 simulated galaxy clusters, containing between 30 and 250 members. The simulations have been created with a maximum of 3 individual structures in a cluster. The simulated clusters are arranged in such a way that $20 \%$ of the sample are clusters with only 1 structure (without substructure), $40 \%$ of the clusters have 
two structures (a primary and a secondary component) and the remaining $40 \%$ of the clusters sample have 3 structures, a primary component, a secondary component and a tertiary component. Thus, $80 \%$ of the simulated clusters show more than one structure. The number of galaxies in each structure is randomized, imposing the condition that the secondary structure is poorer than the primary and richer than the tertiary. For simplicity, the spatial distribution of galaxies in each structure follows a circularly symmetric radial density profile given by $N(R)=N_{0} /\left(1+\left(R / R_{C}^{2}\right)\right.$ ) (an analytic approximation to the King 1962, profile). The core radius and velocity dispersion of each structure are scaled to its number of members. So that there is no overlap between the structures centroids in the position space, the centroid offsets between the primary and every other component are obtained through a uniform random distribution within $\left(R_{C 1}, 4 R_{C 1}\right)$; we do not allow for completely aligned structures along the line of sight because LocKE, just like the usual $\kappa$-test and $\delta$-test, only detects substructures when the velocity distribution and the galaxy projected positions are simultaneously distinct from those of the remaining galaxies in the cluster. The relative velocities between the primary and other structures are randomized and limited to $900 \mathrm{~km} / \mathrm{s}$. The velocity distribution of each structure is assumed to be Gaussian. We run the two algorithms, Mclust and LocKE, on the whole simulated sample.

The frequency of substructures found by Mclust (66\%) was similar to that found by LocKE (63\%); however Mclust found more clusters with 4 or more levels of structures than LocKE, as seen in the histograms in Figure 4. Both methods fail to completely identify the simulated substructures. It was expected that the detection efficiency would not be $100 \%$ for both codes because, for any algorithm, it is not an easy task to find a substructure when its kinematic parameters are comparable to those of the primary component, and this difficulty is still more pronounced when the structures are much poorer than the primary. This difficulty in reproducing the simulated data can be seen in the histograms of Figure 4. Around $50 \%$ of all simulated clusters made up of three kinematical structures are not detected as such - they are mainly classified as a single or a binary cluster but, especially by Mclust, can also be attributed more than the original three structures. We have therefore a combined effect of under-detection of smaller structures and false detection of structures, which actually produce a partial randomization of the detected number of structures with both codes. Regarding the false detection of substructures, however, LocKE does a much better job: among the 624 simulated clusters without substructures, LocKE correctly identified $560(\sim 90 \%)$ as such, while Mclust identified as such only 319 ( $\sim 51 \%)$. We can not define formal completeness limits at this stage, because a successful detection of a substructure involves also the correct identification of their spatial location and kinematics.

A comparison between the simulated and measured properties of each structure is non-trivial. Any detected structure ideally corresponds to a specific simulated structure, but the correspondence between them has to be assessed using some kind of metric. One could use the number of members in each structure, or the kinematic parameters, or a combination thereof. For simplicity, we have chosen an euclidean metric in the mean radial velocity - velocity dis- persion space. Detected structures have been assigned to the simulated ones by minimizing this metric using for the mean radial velocity of the detected structure twice the weight of its velocity dispersion. This is a convenient metric, for it depends only in parameters which are part of the standard LocKE output; we have also tested other, more complex metrics including the number of galaxies per substructure and the substructure centroids, and the results have not changed significantly, so that the discussion below is robust.

The comparison between the simulated and the measured number of members attributed to each structure by the two methods can be seen in Figure 5. For primaries, LocKE presents a tendency for overestimating the number of neighbours. This is mainly an effect of incompleteness when detecting all substructures present. For secondary and tertiary structures, the bias in the number of members is much lower, but the dispersion around the correlation is larger. The Spearman correlation coefficient for primary, secondary and tertiary structures are $0.63,0.57$ and 0.47 respectively. Regarding the slope of these correlations, a Huber robust linear regression (the black dashed lines in Figure 5) performed with the SKLEARN.LINEAR_MODEL package results in slopes of $0.81,0.68$ and 0.58 for primaries, secondaries and tertiaries respectively, confirming that the correlation is close to the 1:1 relation for primaries, but less so to smaller structures. For Mclust, primaries display a bimodal distribution which is not seen in the LOCKE results. A "wing" of fewer detected members can be seen, suggesting that a significant number of galaxies in the primaries are lost to smaller structures. Also, the dispersion in the distributions are consistently higher. The Spearman correlation coefficients for primaries, secondaries and tertiaries, with Mclust, are 0.47, 0.44 and 0.44 , and the slopes of these distributions are 0.30, 0.41 and 0.63. Notice that, in Figure 5, we display only the results for simulated clusters with more than one structure, because the number of galaxies detected will be, by construction, the same as the input number for this kind of simulated cluster. For simulated clusters without substructures and for which LOCKE or Mclust detected at least one substructure, we have calculated the fraction of galaxies which are correctly assigned to the main (richest) structure in the cluster. In this situation, Mclust recovers $63 \%$ of the number of galaxies in the main component, while LocKE presents a much higher rate of $83 \%$.

In Figure 6 we present the comparison between the simulated and measured velocity dispersion using both algorithms. For primaries, both codes perform comparably well; the Spearman correlation coefficient are 0.92 and 0.90 for LocKE and Mclust respectively, and the respective slopes are 0.90 and 0.93 . However, for secondaries and tertiaries, Mclust introduces a very strong bias in the sense of overestimating the simulated velocity dispersion of the substructures. This is a result from the same contamination by galaxies in the primary component which produces the upper "wing" in Figure 5. LocKE, on the other hand, does not bias the velocity dispersion estimates for secondaries and tertiaries. The Spearman correlation coefficients for secondaries and tertiaries are 0.75 and 0.77 for LOCKE and 0.71 and 0.65 for Mclust. The slopes of the correlation for secondaries with LocKE and Mclust are 0.76 and 0.85 , but the intercept is much larger for Mclust $(198.5 \mathrm{~km} / \mathrm{s})$ than for LocKE $(42.9 \mathrm{~km} / \mathrm{s})$. A similar behavior is observed for tertiaries - 


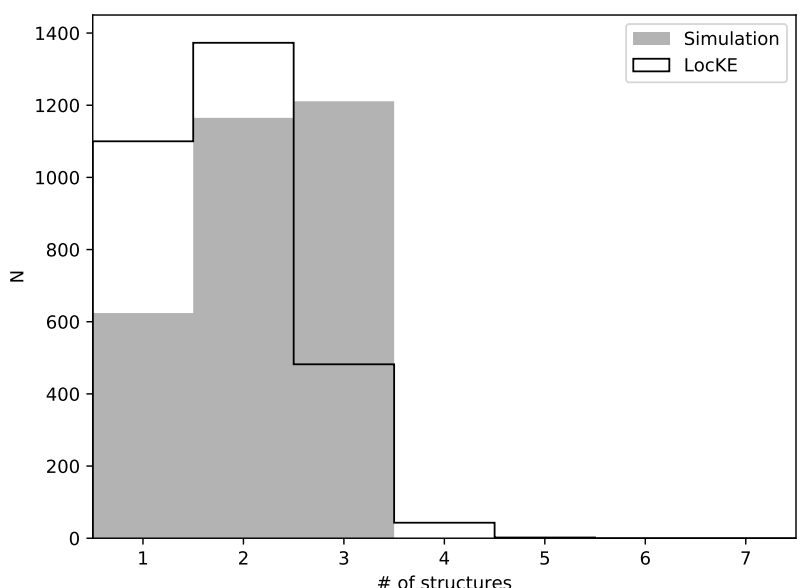

(a)

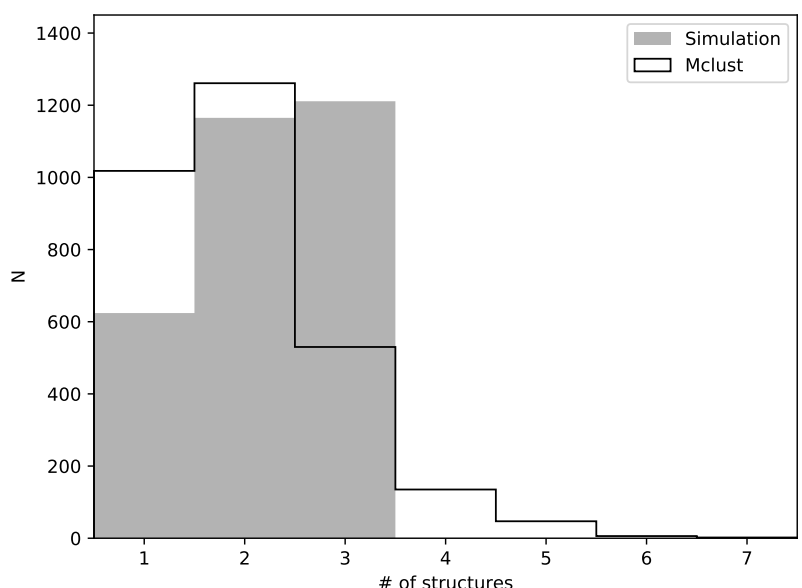

(b)

Figure 4. Comparison between the distributions of the input number of structures in the simulated clusters and the number of structures detected by LOCKE and Mclust. The vertical axes display the absolute number $(N)$ of simulations with one, two or three substructures.
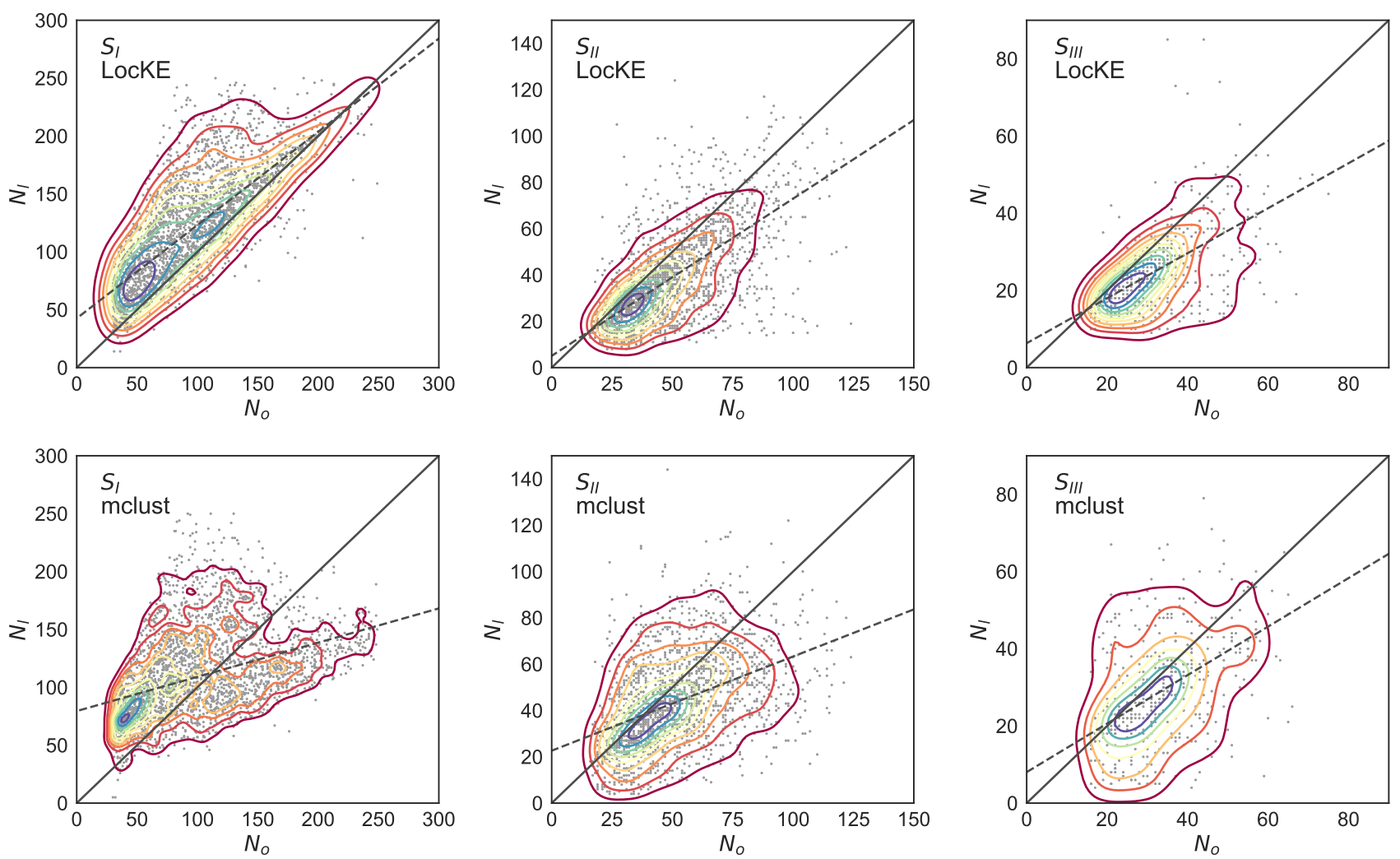

Figure 5. Comparison between the simulated number of members in each structure and those derived by LocKE and Mclust, for primaries $\left(S_{I}\right)$, secondaries $\left(S_{I I}\right)$ and tertiaries $\left(S_{I I I}\right)$. The vertical axes display the input number of members in each structure, $N_{I}$, and the horizontal axes, the detected number of members $N_{o}$ with each algorithm. Individual structures are represented by gray dots, and the local density of points is represented by contours. The black dashed lines represent the best-fit robust linear regression (see text for details). 
slope 0.81 (1.11) and intercept $19.9 \mathrm{~km} / \mathrm{s}(126.8 \mathrm{~km} / \mathrm{s})$ for LocKE (Mclust).

Finally, we show in Figure 7 the mean line-of-sight velocities of the simulated and detected structures. For primaries, the correlation is excellent for both methods (Spearman correlation coefficients of 0.95 and 0.93 for LOCKE and Mclust respectively) and no significant bias is observed. The performance of Mclust for secondaries and tertiaries, however, shows a pronounced bimodality: a family of solutions defining the bi-sector of the correlation plot, plus a less steep, nearly-horizontal distribution. This behaviour is once again due to contamination from galaxies in the primary structure, that tend to reduce the relative line-of-sight velocities (defined as zero in the reference of the primary). LocKE on the other hand presents a better behaved relation, with some scattering outside the bi-sector and mostly due to a wrong assignment of the substructure level (primary/secondary). The correlation coefficients for secondaries and tertiaries are 0.66 and 0.60 for LocKE and 0.74 and 0.52 for Mclust. (Notice that, even though the correlation coefficient for secondaries is larger for Mclust than for LocKE, the slope of the correlation is strongly biased.)

We therefore conclude that LocKE, even though presenting the intrinsic weaknesses of the $\delta$ and $\kappa$ tests (the inability of identifying substructures aligned in the line of sight), it does a good job at detecting and estimating the kinematic properties of substructures, and also in isolating their members. Though introducing some level of bias in these parameters, particularly due to undetected structures, they are much better behaved than multidimensional mixture as modelled by Mclust, while maintaining its main strength of being fully automated. We have used LocKE to investigate the presence of substructures in our sample 412 selected clusters of galaxies and present the results in Sect. 4.1.

\subsection{Stellar population synthesis}

To investigate the influence of the dynamic stage of the cluster in the stellar populations of galaxies, we performed stellar population synthesis and derived the mean stellar age of each galaxy in the sample. For this, we made use of the STARLight code (Cid et al. 2005; Mateus et al. 2006). As templates we have used single stellar population models (SSPs) from the Medium resolution Isaac Newton telescope Library of Empirical Spectra (MILES) (Sanchez et al. 2006), which match roughly the SDSS spectral resolution $(\sim 2.3 \AA)$ and cover the optical range from 3525 to $7500 \AA$. We have selected a set of 156 SSPs with 26 different ages ranging from $70.8 \mathrm{Myr}$ up to $14.12 \mathrm{Gyr}$, and 6 different metallicities ranging from $Z=0.0004$ up to $Z=0.0315$ (i.e. from $Z=0.02 Z$. to $\left.Z=1.575 Z_{\odot}\right)$. Prior to the synthesis, the observed SDSS spectra have been converted to linear scale, resampled to steps of $1 \AA$, corrected for Galactic extinction and corrected to restframe with the Python code PYSTARLIGHT ${ }^{2}$.

The Starlight output includes the mass and light con-

2 Available to download at http://www.starlight.ufsc.br/node/3 tribution of each SSP to the observed spectrum. The lightweighted mean stellar age $\bar{t}$ of a galaxy is defined by

$\log \bar{t}=\sum_{j=1}^{156} \log t_{j} L_{j} / \sum_{j=1}^{156} L_{j}$,

where $t_{j}$ is the age of template $j$ and $L_{j}$ is its contribution to the observed spectrum. We have also derived the stellar masses, in units of the solar mass, using

$M_{\star}=\frac{M_{\text {cor_tot }} \times 10^{-17} \times 4 \pi d l^{2}}{L_{\odot}}$,

where $M_{\text {cor_tot }}$ is a STARLIGHT output, $d l$ is the luminosity distance, and $L_{\odot}$ is the solar luminosity. We then correct the fiber stellar mass obtained above to total stellar mass by scaling the total and fiber $r$-band magnitudes, resulting in an increase of $4 \%$ on average relative to the fiber stellar masses.

\section{RESULTS}

\subsection{Frequency of substructures}

For the full sample of 408 clusters presenting more than 30 members, LocKE detected substructures in 180 , or $\approx$ $45 \%$ of the sample. This value is in agreement with typical frequencies using a single method for substructure detection. Aguerri \& Sánchez-Janssen (2010), for example, using the $\delta$ test, have found substructures in $34 \%$ of their sample of 88 rich galaxy clusters. Solanes et al. (1999) studied a sample of 67 rich clusters extracted from ENACS catalog using four different statistical tests. A normality test indicated that $30 \%$ of the sample are unrelaxed, and with the $\delta$-test they found a frequency of substructures of $31 \%$. Einasto et al. (2012), on the other hand, adopted multiple 1-D (ShapiroWilk and Anderson-Darling), 2-D ( $\beta$-test) and 3-D ( $\alpha$-test, $\delta$-test and multidimensional mixture modelling with Mclust) to investigate 109 clusters of galaxies, and found a large frequency of $70 \%(80 \%)$ of clusters with substructures as indicated by the $\delta$ test (Mclust).

For further analysis, we will split the sample clusters in three broad categories. Clusters with no substructures detected will be referred to as "no substructures" and represented by NS. For clusters presenting substructures, we will refer separately to the main structure, or main halo, defined as the most massive structure in the cluster (represented by PC, or "Primary Component") and all lower mass structures as "secondaries" no matter the number of structures detected (hereafter, SC or "Secondary Component"). We will also refer to the full population of galaxies in clusters presenting substructures, before separation in individual units by LocKE, as "unrelaxed", and represent them by the symbol U, when convenient.

\subsection{Properties of the individual kinematic components}

After the identification of kinematic substructures, LocKE provides kinematic parameters for each structure. Of particular importance is the velocity dispersion, that is used to 

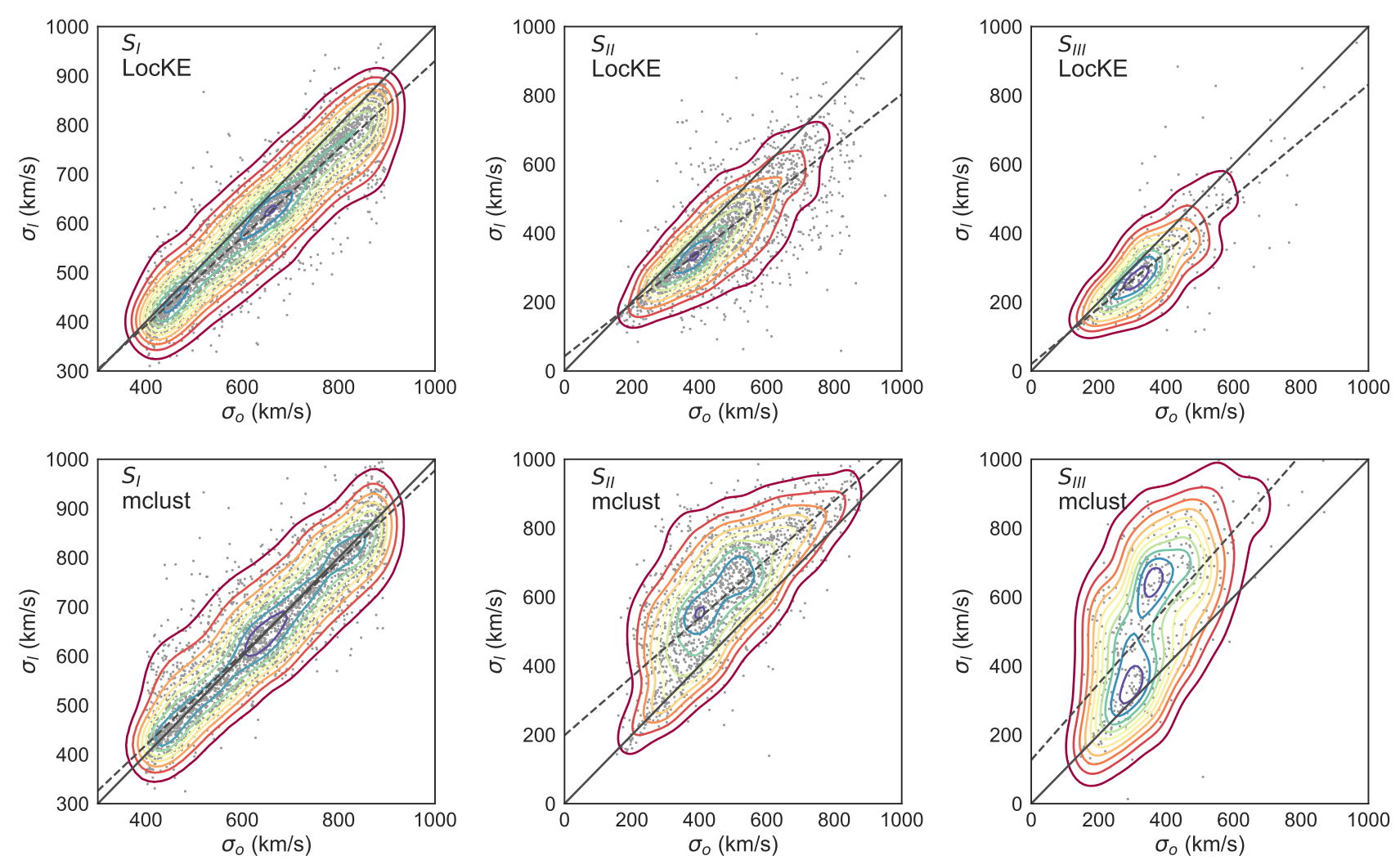

Figure 6. Comparison between the simulated velocity dispersion and those measured with LocKE and Mclust, for primaries $\left(S_{I}\right)$, secondaries $\left(S_{I I}\right)$ and tertiaries $\left(S_{I I I}\right)$. Symbols are the same as in Figure 5.

calculate the virial mass. In Figure 9 we show the velocity dispersion distribution of all structures found in our sample. The blue, red and green curves represent NS, PC and $\mathrm{SC}$ clusters; the mean velocity dispersion for each class is $400 \pm 76 \mathrm{~km} / \mathrm{s}, 417 \pm 96 \mathrm{~km} / \mathrm{s}$ and $231 \pm 81 \mathrm{~km} / \mathrm{s}$. These values are typical of medium-sized clusters and groups (Colles \& Dunn 1996; Aguerri \& Sánchez-Janssen 2010; Hou et al. 2012 , e.g.).

Figure 10 presents the mass distribution of all structures of the sample as derived by LocKE. The mean values for the logarithmic virial mass are 14.19, 14.1713 .34 dex for NS, PC and SC, respectively, which are also in agreement with typical values for this class of object (Carlberg et al. 1996; Lopes et al. 2009, e.g.). Regarding the physical size of each structure, we have found $R_{200}$ values of $0.96 \mathrm{Mpc}$, $1.00 \mathrm{Mpc}$ and $0.55 \mathrm{Mpc}$ for $\mathrm{NS}, \mathrm{PC}$ and $\mathrm{SC}$, respectively. The distribution of this parameter is shown in Figure 11, also in agreement with other authors (e.g. Carlberg et al. 1997; Lopes et al. 2009; Yan et al. 2015).

The above distributions show that primary structures (PC) are comparable in mass and radius to the NS class. These two classes are therefore physically similar, with the exception that the former are associated to other nearby structures. Secondaries, on the other hand, are consistently less massive, and smaller in size than PC and NS.

\subsection{Stellar populations in individual structures}

In this section we investigate how the typical mean stellar ages of galaxies in our sample respond to the class of structure they populate. Figure 12 presents the cumulative distributions of the light-weighted logarithmic mean stellar age of galaxies in each structure class. The thickness of the curves indicate the poisson errors. We have chosen to present the cumulative distributions instead of a discrete histogram in order to make the differences more clear. The age distribution of relaxed (NS) versus unrelaxed (U) clusters are distinct, with unrelaxed clusters (in black) presenting a larger contribution of galaxies with younger stellar populations. When dividing $U$ clusters into primaries and secondaries, the behavior of the mean stellar age is quite similar among the three classes, with some subtle differences. Galaxies located in the secondaries (SC; green curve) tend to present younger stellar populations. For galaxies in the primaries (PC; red curve) ages tend to be slightly lower than for NS (blue curve) clusters, but the difference is very small. This means that, when excluding the lower mass substructures from unrelaxed clusters, the resulting age distribution becomes much closer to the relaxed cluster sample.

Trying to clarify these differences in mean stellar age, we now investigate how these depend on galaxy stellar mass. In Figure 13 we present the mean stellar ages for our three classes of structures as a function of their stellar mass. The error bars have been obtained via bootstrap resampling with 

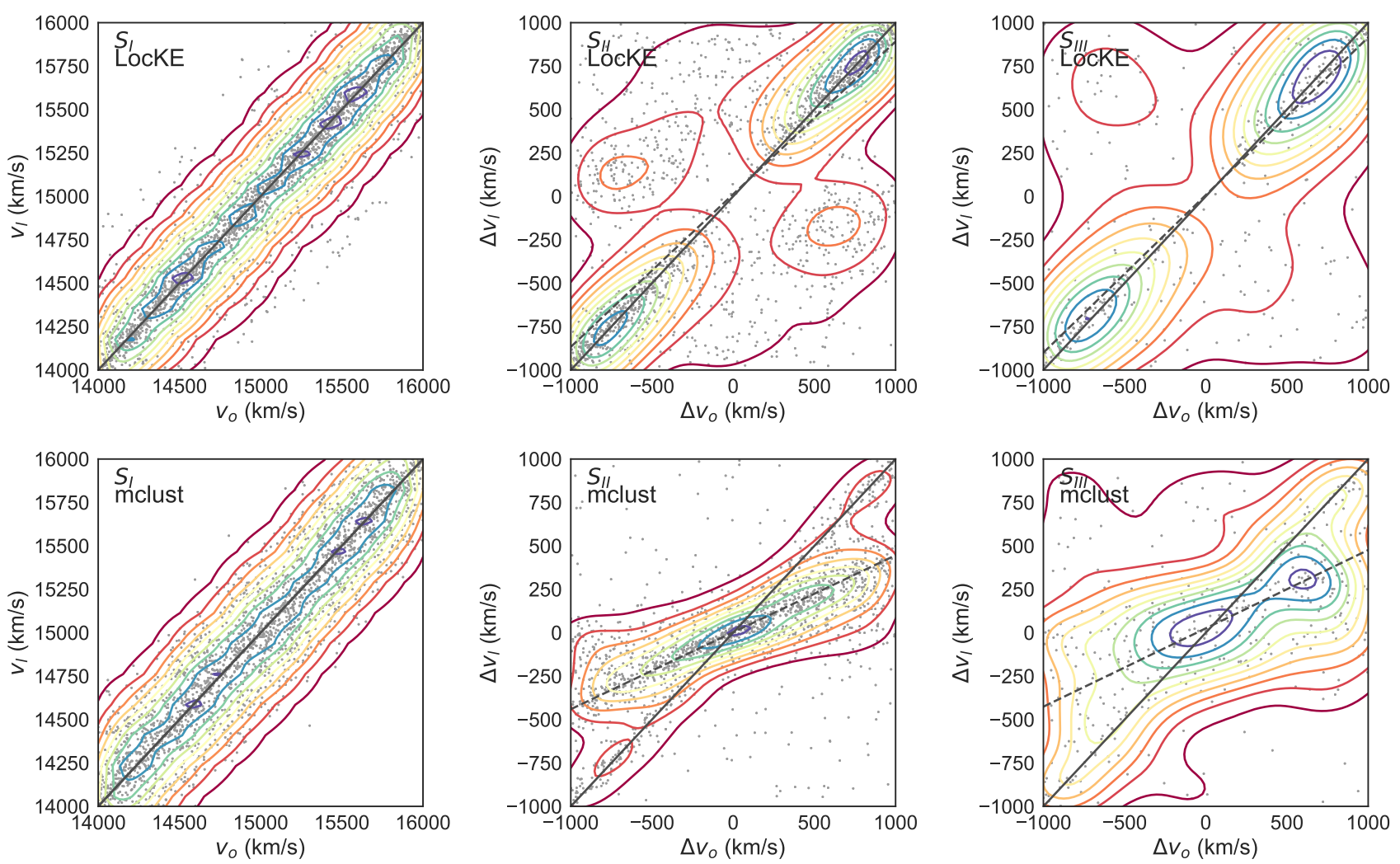

Figure 7. Comparison between the simulated mean line of sight velocity and those measured with LocKE and Mclust, for primaries $\left(S_{I}\right)$, secondaries $\left(S_{I I}\right)$ and tertiaries $\left(S_{I I I}\right)$. Symbols are the same as in Figure 5 .

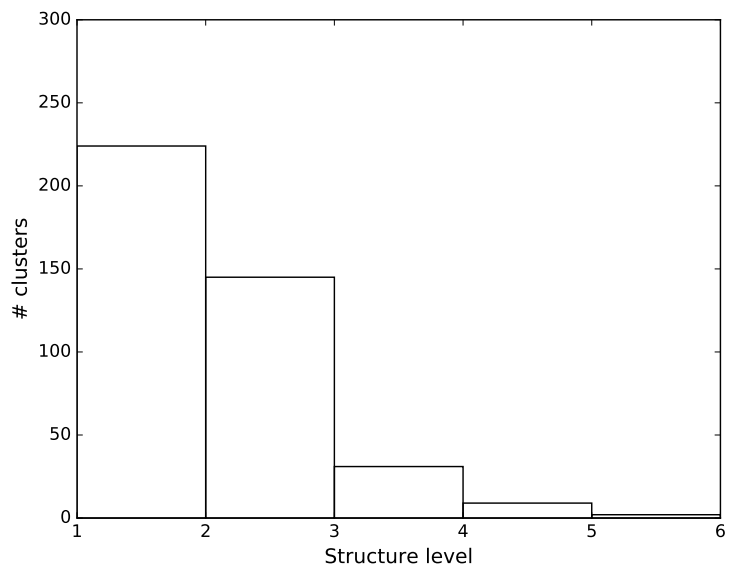

Figure 8. Number of clusters of galaxies in our sample as a function of the number of kinematic structures identified by LocKE.

10,000 realizations. The most evident feature of this figure is that galaxies with higher stellar mass are older for all classes, including field galaxies (yellow curve). This reflects the well-established phenomenon of downsizing, in which the star formation history is skewed towards lower redshifts for less massive galaxies (Neistein et al. 2006; Fontanot et al.

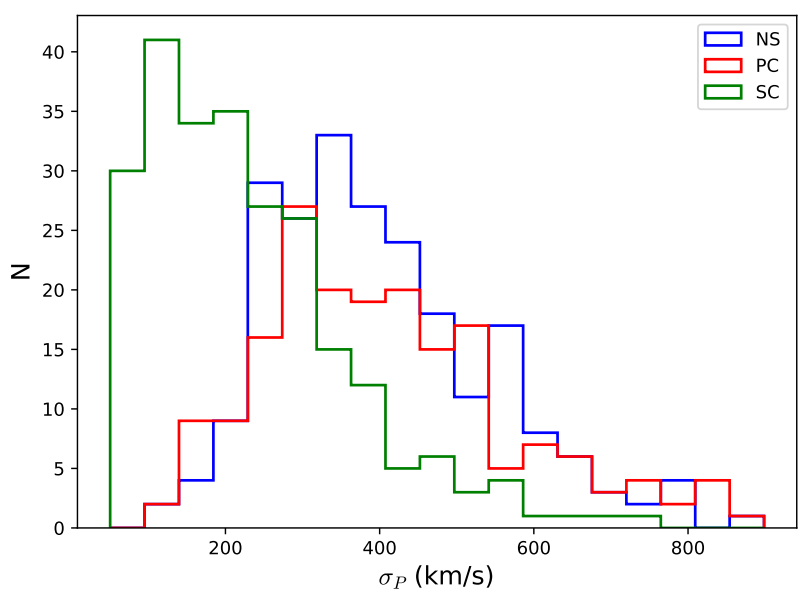

Figure 9. Velocity dispersion distribution for clusters without substructure (NS), primary components (PC) and secondary component (SC).

2009). We also can see that galaxies in secondary structures (SC) seem to present lower mean ages relative to primary components (PC) and clusters with no substructure (NS). These differences are very small - corresponding to less than $1 \mathrm{Gyr}$ in mean stellar age - and stronger at masses lower than 


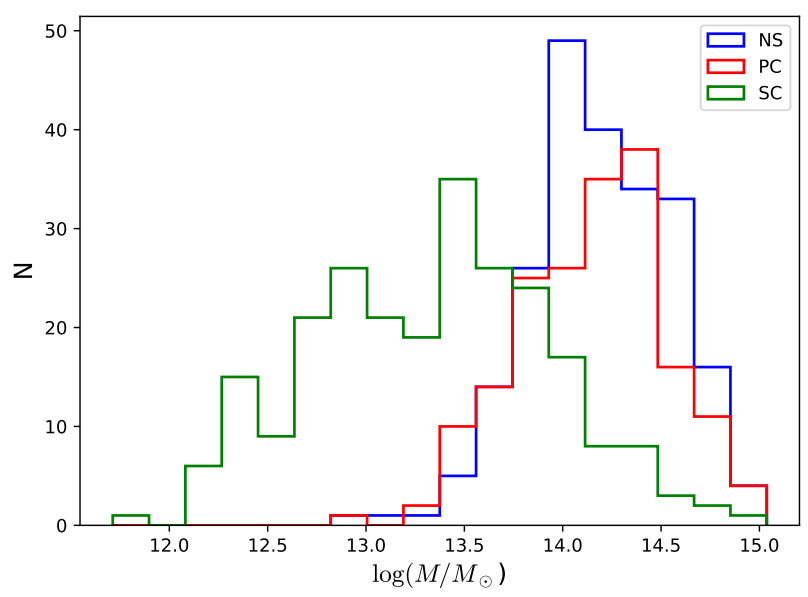

Figure 10. Mass distributions for NS, PC and SC structures of the clusters in our sample. Masses of PC and NS structures are similar, and larger than the typical masses of the secondary components.

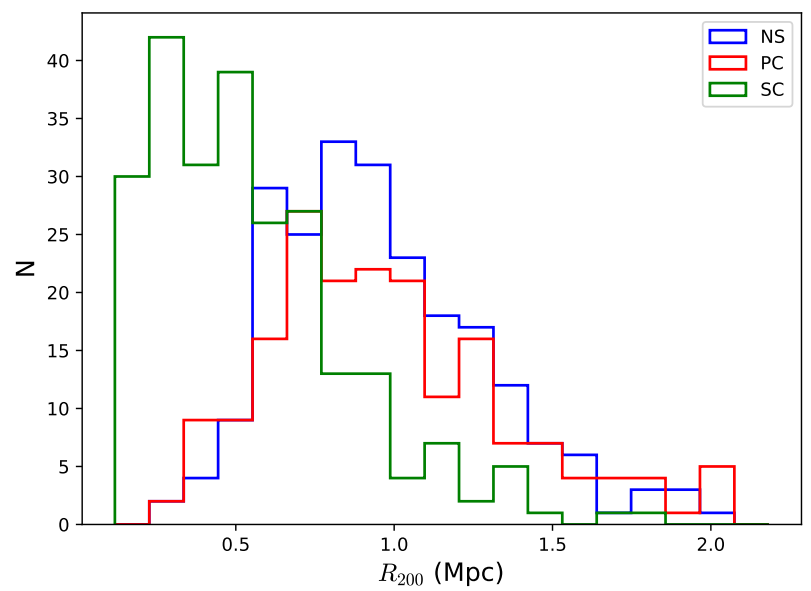

Figure 11. Distribution of $R_{200}$ values for all structures in our sample, showing similar sizes for NS and PC structures; SC structures tend to be much smaller.

$10^{10.8} \odot$. At the lowest mass bins $\left(\lesssim 10^{9} \odot\right)$, the difference is less clear due to the large statistical noise. Galaxies in $\mathrm{PC}$ also tend to present younger stellar populations relative to NS clusters, but the difference is lower than for SC at all mass bins. Field galaxies are younger, for all mass bins, than galaxies in any cluster structure, and this difference can reach up to $\sim 3 \mathrm{Gyr}$.

We now investigate how the mean stellar age of galaxies respond to their clustercentric distance. In Figure 14 we present the mean stellar age as a function of the galaxy clustercentric distance normalized to $R_{200}$, separating the sample according to the three structure classes. We notice an overall tendency of lower mean stellar ages occur at large clustercentric distances for the three classes. This result reflects the well-established relationship between galaxy properties (morphology, age, SF, etc.) and clustercentric distance (e.g. Dressler 1980; Kauffmann et al. 2004; Blanton et al. 2005). However, this radial mean age gradient can be seen for all magnitude ranges and structure classes, so that galaxies closer to the center of every structure present older stellar populations than galaxies in their outskirts, no matter the status of the structure. This shows that some kind of preprocessing has already been acting on the galaxy population which is being accreted into primaries. We can see, however, that the slope of the age-radius relation is consistently less steep for secondaries. For primaries, on the other hand, the age-radius relation seems to be completely in place. Using cosmological N-body simulations, Ludlow et al. (2009), have shown that, during the process of virialization of a cluster after accreting a low-mass galaxy system, the most important parameter defining the orbits of accreted galaxies is the orbital phase coupling between the orbit of the substructure and the main component of the cluster. The dissolution of a substructure is therefore expected to contribute with a new population of galaxies across the full extend of the primary. This in turn implies that a shift in mean stellar age for recently accreted galaxies is expected to occur before the clusters detected as "unrelaxed" attain a new virialization state.

Because mean stellar ages are higher for more massive galaxies, it is important to check whether these radial gradients in mean stellar age are an artifact due to a radial gradient in galaxy stellar mass. We have tested this hypothesis in two complementary ways. Firstly, we substitute the mean stellar age of every cluster member (at any structure level) by the mean stellar age of a field galaxy at the same stellar mass. If the observed radial age gradients are produced by a stellar mass gradient, replacing cluster galaxies by field galaxies of the same stellar mass should reproduce the observed age gradients. The result of this experiment is illustrated by yellow dots in Figure 14. Some degree of dependence of the mean stellar age on clustercentric radius is indeed observed for galaxies drawn from the field population. However, the resulting gradient is much less steep (by a factor $\sim 6$ ) than that observed by cluster galaxies in NS and PC structures, indicating that galaxy stellar masses are not the main driver of the observed radial trends in mean stellar age for these massive structures. For $\mathrm{SC}$ structures, on the other hand, the slope of this relation is not much larger than that for field clusters. In order to be able to perform a better comparison between the slopes of this relation for cluster galaxies and that obtained with field galaxies, we have split the field sample into three subsamples - NSField, PCField and SCField - drawing galaxies following stellar mass distributions similar to each individual structure. We have then performed linear regressions for the derived mean stellar age - clustercentric radius relations for cluster galaxies (NS, PC and SC) and for their corresponding field galaxies (NSField, PCField and SCField). We have obtained the following slopes: $\alpha_{N S}=-0.28 \pm 0.01$, $\alpha_{P C}=-0.27 \pm 0.02, \alpha_{S C}=-0.17 \pm 0.02$ for cluster galaxies, and $\alpha_{N \text { SField }}=-0.08 \pm 0.01, \alpha_{P C \text { Field }}=-0.07 \pm 0.02$, $\alpha_{S C \text { Field }}=-0.03 \pm 0.02$ for field galaxies. The differences between these slopes is $-0.20 \pm 0.02$ for both NS and PC structures, and reduces to $-0.14 \pm 0.03$ for SC structures. Therefore, for all structures, both the stellar age gradients and the "gap" between slopes of SC structures and more 

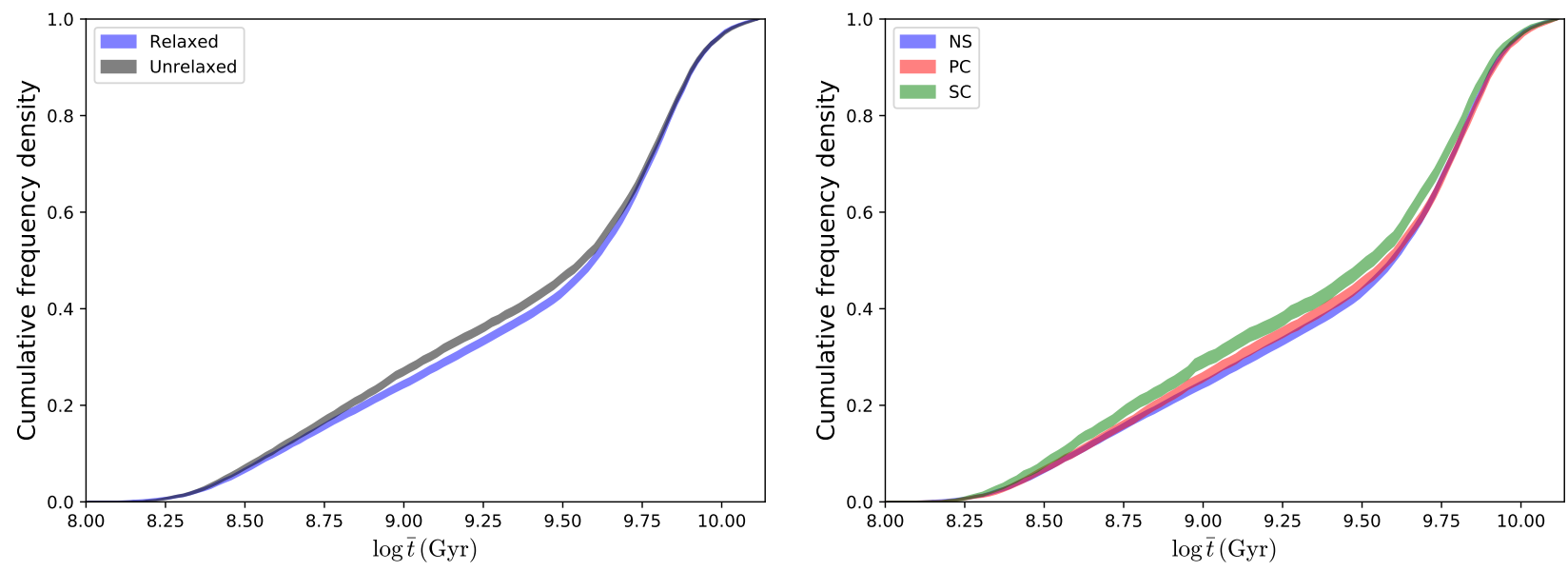

Figure 12. Cumulative frequency of the logarithmic stellar age distribution for galaxies in each structure class defined in this work. In the left panel, relaxed clusters are represented by the blue shade, and unrelaxed (U) clusters are shown in black. In the right panel, the blue, red and green curves represent NS, PC and SC structures, respectively.

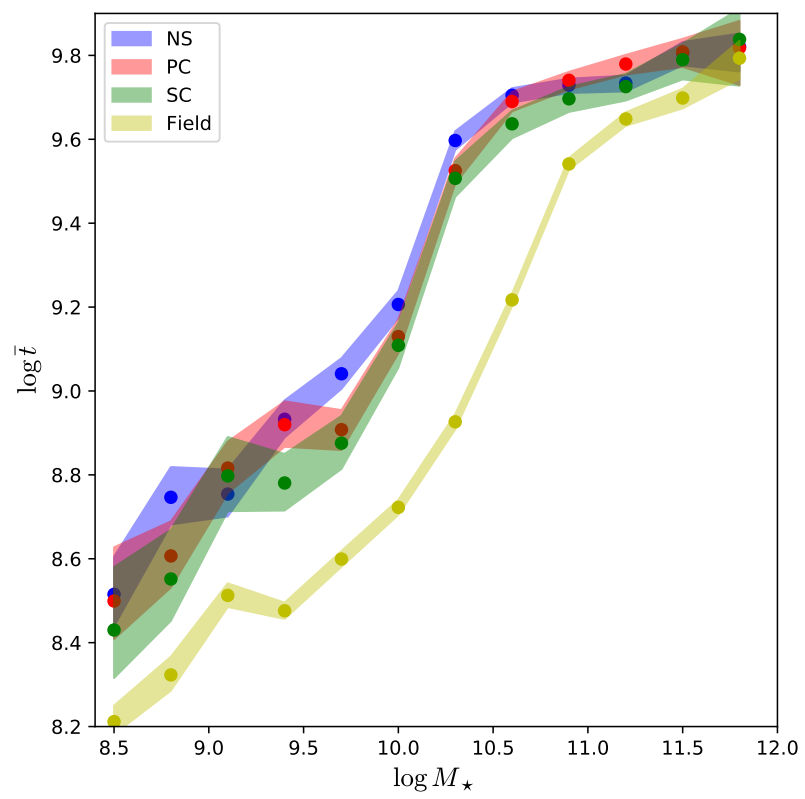

Figure 13. Mean stellar age as a function of stellar mass for galaxies in each of the three structure classes defined in this work and for a sample of field galaxies. The blue, red and green curves represent NS, PC and SC structures, respectively, and yellow curve represent the field sample.

massive ones are shown to be preserved when we take galaxy stellar masses into account.

The second test we have performed is a direct calculation of the mean stellar mass of cluster galaxies as a function of the radial clustercentric distance. This is presented in Figure 15. A radial mass gradient can be detected in our cluster galaxies at all structure classes; in fact, some degree of radial dependence of stellar mass in cluster galaxies has been observed by other authors (e.g., Roberts et al. 2015). However, these gradients are very small - corresponding to less

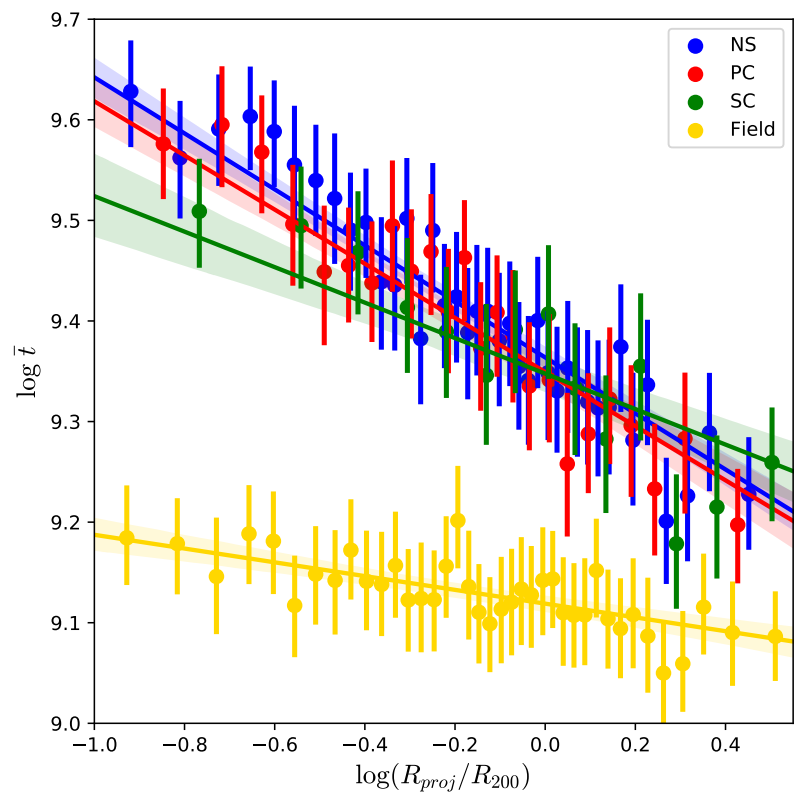

Figure 14. Logarithm of mean stellar age as a function of the distance of the galaxies to the center of its parent structure. Each dot represents the average mean stellar age in bins of radial distance. Blue dots represent relaxed (NS) clusters, red (green) dots stand for primaries (secondaries) of unrelaxed clusters, and yellow dots designate field galaxies. The error bars indicate the standard error of the mean.

than $40 \%$ in average stellar mass across the full radial extension of the structure up to $2.5 R_{200}$ - and, more importantly, stellar masses correlate with the clustercentric radius much worse than the mean stellar age. We measured the Spearman correlation coefficient for mean stellar age and clustercentric distance, obtaining -0.23 for both NS and PC, and -0.17 for SC structures. The Spearman correlation coefficient between stellar mass and clustercentric distance, in contrast, are $-0.09,-0.08$, and -0.06 for $\mathrm{NS}, \mathrm{PC}$ and $\mathrm{SC}$, respec- 


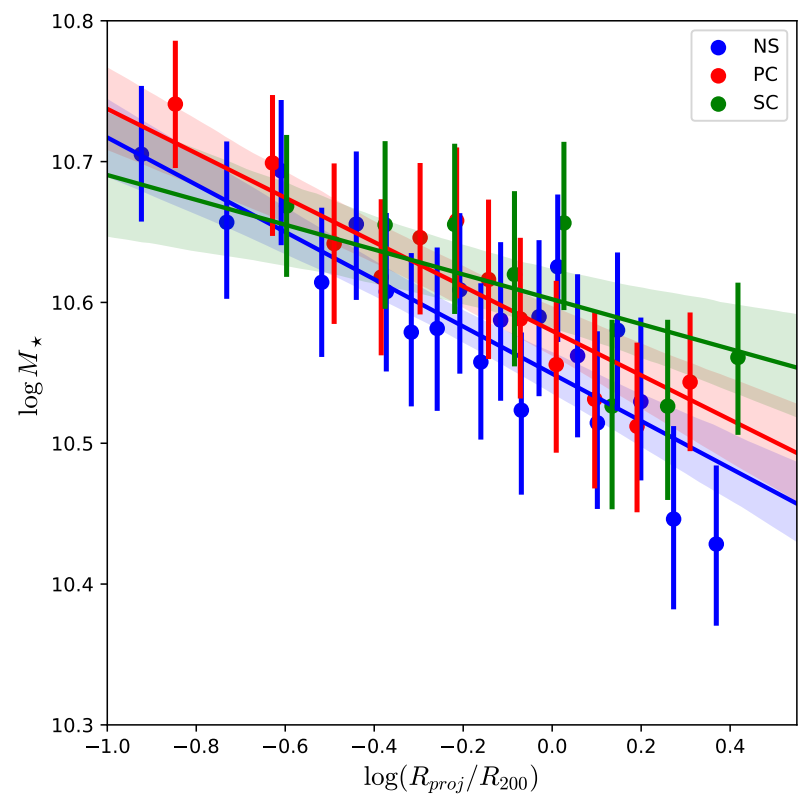

Figure 15. Logarithm of stellar mass as a function of the distance of the galaxies to the center of its parent structure. Each dot represents the average mean stellar age in a bin in radial distance. The dots and colors follow the same configuration as in figure 14 .

tively. It is therefore not possible for these small, disperse clustercentric stellar mass gradients to produce much tighter and steeper mean stellar age gradients.

The results above suggest that the main difference between the stellar populations in individual structures comes mainly from the differences in total halo mass. In order to further explore this possibility, we plot in Figure 16 the mean stellar age of galaxies in each structure class as a function both of the mass and the velocity dispersion of the halo they reside. As in Figure 12, the right column of Figure 16 shows the combination of PC and SC. Before separation between primary and secondary, we see a consistent age difference: Unrelaxed clusters are younger than their relaxed counterparts, even though they share the same mass and velocity dispersion range. When separating the secondaries, the agemass and age-velocity dispersion relations become almost indistinguishable for large mass structures. This suggests that $\mathrm{PC}$ and NS are comparable structures, and that the main differences between clusters in different evolutionary stages (at fixed mass) come from the presence of secondaries.

\section{DISCUSSION}

Using LocKE, we have detected substructures in around $45 \%$ of the clusters in our sample. Interpreting the presence of substructures as a proxy for the dynamical stage of the clusters, this frequency indicates a large proportion of unrelaxed clusters in our sample. Furthermore, the LocKE performance testing implies a family of unrelaxed clusters which our methodology is unable to identify as such. This is due mainly to a combination of alignment of structures in the line of sight. We therefore consider the above figure as a lower limit to the number of unrelaxed clusters in our sample. Contamination from these objects will tend to reduce the differences between the clusters detected as relaxed and unrelaxed.

Estimating the velocity dispersion, masses and virial radius of each structure, we found that relaxed clusters and the primary structures (i.e. the most massive) of unrelaxed clusters share approximately the same parameter distributions. Therefore, for every unrelaxed cluster in the sample, there exists a dominant mass structure which is comparable in mass and size to a relaxed cluster in the sample. Secondaries on the other hand are consistently less massive and smaller. This means that the main source of unrelaxed clusters in our methodology is the occurrence of low-mass, small systems around massive structures.

The analysis of the mean stellar ages obtained by a stellar population synthesis using STARLIGHT resulted in different age distributions for relaxed and unrelaxed clusters, with a larger contribution of younger stellar populations in unrelaxed clusters with relation to relaxed ones. This result is in accordance with the findings of Ribeiro, Lopes \& Rembold (2013), though these authors have used a onedimensional test to evaluate the dynamical stage of the clusters in their sample. Our findings are also in accordance to Lopes et al. (2014), which have found a larger proportion of blue galaxies in the outskirts of unrelaxed clusters when compared to relaxed ones.

We then obtained the mean age distributions separating unrelaxed clusters into primaries and secondaries. The resulting mean age difference relative to relaxed clusters are more evident for secondaries, while primaries are much more similar to relaxed clusters. Note that we are not suggesting, at this stage, that the status as "secondary" has any physical implication except the fact that it is probably contained in the sphere of influence of a larger cluster system. This being the case, the evolution of the whole system will proceed and eventually reach a new equilibrium state. We have found that the main mass component of a relaxed cluster presents a distribution of stellar populations which is similar to that of relaxed clusters in the same mass ranges. This is not true of secondaries, and this difference may be derived from the physical conditions the status as "secondary" induces in the structure or simply by the fact that the other global parameters are diverse (e.g. mass and radius).

The differences we have found in the age distributions of relaxed and unrelaxed clusters are mainly associated to low-luminosity galaxies. Galaxies above $\sim 10^{10.8} M_{\odot}$ do not show any detectable sensitivity to the environment in what regards the mean stellar ages of the galaxies therein. This result is also in accordance with the literature. A possible explanation for this effect is a scenario where the environment, during cluster virialization, quenches the star formation in low-mass galaxies. Indeed, Wu et al. (2014) have found that in dynamically young clusters the star formation rate is higher than in evolved clusters. Furthermore, Peng et al. (2010) have found that the stellar mass range $\log M / M \odot \lesssim 10.5$ dex correspond to the range where the environment is the main driver of quenching. Therefore, unrelaxed clusters present lower mean stellar ages because they are richer in young low-luminosity objects, which have still not undergone the stellar population evolution characteristic of evolved systems. Because the main age differences are 

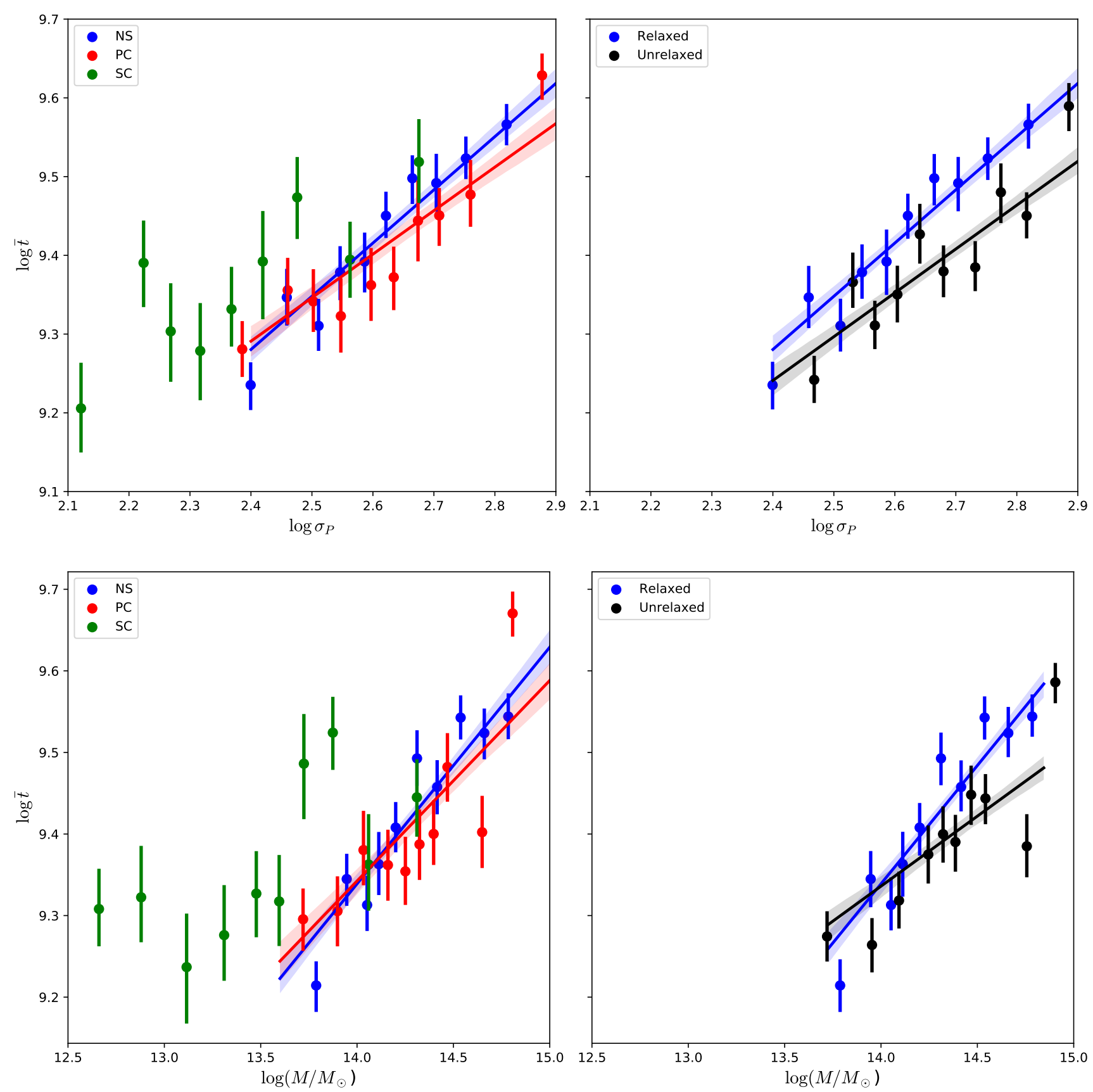

Figure 16. Logarithm of mean stellar age as a function of the halo velocity dispersion (top) and halo mass (bottom). Symbols in the left plots are the same as in Figure 14. In the right plots, relaxed clusters (NS) are represented by blue dots, and black dots indicate unrelaxed (U) clusters.

found for secondaries, this suggests that these peripheral, low-mass systems bring galaxies which are not representative of the environment of the primary, massive components.

Once there exists an age distribution which is specific for secondaries, how do these objects distribute in the body of the structure they reside? We have determined how the mean stellar ages vary as a function of the center of the structure for the three structure classes. We have found an age gradient well established for all classes, but less intense in the secondaries. At large radii, the mean ages are similarly low between all classes. In the central parts, however, secondaries are consistently younger. We therefore conclude that galaxies in secondaries are already partially pre-processed. The existence of well-defined age-clustercentric distance relations for both relaxed and unrelaxed clusters is not new (e.g. Ribeiro, Lopes \& Rembold 2013), but we here show that the pre-processing in secondaries is not as advanced as in the primaries or in relaxed clusters. A comparable result 
has been found by Olave-Rojas et al. (2018) for two massive clusters. These authors have found that the fraction of red galaxies is higher in central regions of both clusters and substructures therein, and decrease with clustercentric distance. The authors also measured the environmental quenching efficiency, and determined that this efficiency is higher in the central regions of all structures, but particulary in the cluster inner regions. This difference may be due to the environmental processes that depend on the high density found in the central region of massive clusters, like ram-pressure or strangulation. The difference we have found in mean ages for primaries and secondaries, in the central regions, is low $(\sim$ 1 Gyr), so that any evolutionary effects with a comparable timescale ( 1-2 Gyrs) can erase this difference.

The findings above suggest that the mass of the structure is the major parameter behind the mean stellar ages of its galaxy populations. We have tested this hypothesis verifying how the mean stellar age depends on the velocity dispersion and the mass of the structure, both before and after the decomposition of unrelaxed clusters into primaries and secondaries. We have found age-velocity dispersion and age-mass relations well established for relaxed and unrelaxed clusters, but these relations are distinct for these two classes. By and large, unrelaxed clusters present lower mean ages than relaxed clusters in the same range of mass and velocity dispersion (with the possible exception of the low- mass limit). This is in contrast with the findings of Lopes et al. (2014), which have found no major impact of the parent halo mass on the distribution of the galaxy colors. The difference we have found is more evident in velocity dispersion, which is more easily contaminated by the presence of substructures, but is visible also in terms of the system mass. When we separate primaries and secondaries, we see that primaries present relations almost indistinguishable from relaxed clusters. Secondaries in general present mean ages close to the lower limit for relaxed clusters, with some contamination of very old structures in the high-mass regime, probably accounted for by an incorrect separation of components by LocKE. This reinforces the idea that primaries and relaxed clusters are similar and comparable structures, obeying the same kinematic scaling relations. We have shown that the secondary population is partially pre-processed, in accordance e.g. to de Carvalho (2017), but this evolution is incomplete, producing most of the observed differences in mean stellar age distribution and radial clustercentric gradient. These findings suggests that, as the unrelaxed clusters evolve, galaxies being accreted in the form of the secondary population will evolve due to specific mechanisms operating in the high-density of the primaries.

The exact mechanisms by which this evolution occur are uncertain. By means of N-body plus hidrodynamical simulations of a merge between a group-size structure and a cluster of galaxies, Vijayaraghavan \& Ricker (2013) have shown that both pre-processing (due to enhanced galaxygalaxy merger rates, ram pressure stripping in the group environment, and tidal stripping) and post-processing (due to enhanced galaxy-galaxy collisions and/or tidal stripping driven by the density enhancement near the pericentric passage as well as an increased ram pressure along the shock front) can be important for producing the stellar population distribution of cluster galaxies after the cluster virialization. This combination of pre- and post-processing has also been invoked by e.g. Taranu et al. (2014). These authors have found that a combination of quenching in lower mass groups plus quenching in the cluster environment best describes the observed colors and absorption indices of cluster galaxies when combined with a library of subhalo orbits from N-body cosmological simulations, favouring quenching processes with long (such as strangulation) over short timescale processes (such as ram pressure stripping). These works agree qualitatively with our findings in the sense that radial age gradients are ubiquitous in our sample of low-mass secondaries, pointing to some degree of pre-processing, but both the slope of this radial gradient and the mean stellar age of galaxies therein are lower than in relaxed clusters, indicating that further evolution has to operate after the parent haloes merge. On the other hand, de Lucia (2012), comparing galaxy merger trees with optical colors of group and cluster galaxies, have found that more massive haloes present a higher fraction of quenched galaxies due to the fact that satellites of massive haloes have been satellites - and therefore subject to environmental quenching - for a longer time than satellites of low-mass haloes. As a consequence, the fraction of quenched galaxies must scale with the parent halo mass. This helps to explain the correlation we have found between the structure mass and mean stellar age for relaxed clusters and primaries. However, this would imply that the observed differences between the stellar populations of secondary structures and relaxed clusters is not due to an increased quenching efficiency in high mass systems - i.e. quenching could proceed for satellite galaxies at the same rate as before the merging. Both scenarios - including or not an increased post-processing efficiency - could be reconciled with our results due to the small differences we have found in mean stellar ages, as long as the scaling relations after unrelaxed cluster virialization evolve quickly enough to match those of relaxed clusters. A direct test of the occurrence of increased post-processing in our sample can be made by investigating the properties of galaxies in unrelaxed clusters as a function of their projected position with relation to the putative shock front and the evolutive stage of the merge. This analysis will be presented in a forthcoming paper.

\section{CONCLUSIONS}

We have investigated the occurrence of unrelaxed systems in a sample of 408 clusters of galaxies drawn from the Tempel et al. (2012) catalogue. For substructure detection, extraction and automatic estimation of the kinematic parameters we devised the code LocKE (Local Kinematic Estimator) and tested its performance against another multidimensional decomposition code used to detect substructures in clusters of galaxies (mclust). The individual structures in unrelaxed clusters have been separated into primaries and secondaries according to their dynamical mass. The mean stellar ages of galaxies in relaxed and unrelaxed clusters have been derived using the results of a stellar population synthesis with the STARLIGHT code. Our main findings can be summarized as follows:

- We found a frequency of $\sim 45 \%$ of substructures in the sample clusters, which is in agreement with the literature. Given the intrinsic incompleteness of LocKE for structures 
aligned with the line of sight, this figure must be seen as as upper limit to the real frequency of unrelaxed clusters in the sample.

- Primary (i.e. the most massive) structures in unrelaxed clusters present a similar mass distribution as relaxed clusters, while secondaries are much smaller and less massive.

- In unrelaxed clusters, the galaxies present a lower mean stellar age than galaxies in relaxed clusters. When separating unrelaxed clusters into primaries and secondaries, we find that most of this difference is due to lower mean stellar ages in secondaries.

- The age differences above are detected for all galaxy stellar masses, but seem to be less marked at the highest stellar mass bins ( $\gtrsim 10.8 \mathrm{dex}$ ).

- A mean age-radius relation is observed both for relaxed clusters and for primaries and secondaries in unrelaxed clusters. The slope of the relation is, however, less steep for secondaries. This suggests that substructures in clusters bring to the system galaxies which are already pre-processed at the group scale, but this pre-processing is still incomplete and will proceed after the galaxies penetrate into the dense regions of the primaries.

- Relaxed and unrelaxed clusters describe different mean age - mass and mean age - velocity dispersion relations. However, the same relations are nearly identical for primaries and relaxed clusters. We interpret our findings as an evidence that the large-scale merging of galaxy systems does not have a dramatic impact on the stellar populations of galaxies in primary substructures. On the contrary, the observed trends can be explained as a result of the infall, onto large systems, of low-mass systems which are, due to its reduced mass, richer in galaxies with younger stellar populations. The virialization process of unrelaxed clusters is therefore accompanied by the evolution of the stellar populations of galaxies in these infalling groups.

\section{ACKNOWLEDGEMENTS}

We thank FAPERGS and CNPq for financial support. This study was financed in part by the Coordenação de Aperfeiçoamento de Pessoal de Nível Superior - Brasil (CAPES) - Finance Code 001.

Funding for SDSS-III has been provided by the Alfred P. Sloan Foundation, the Participating Institutions, the National Science Foundation, and the U.S. Department of Energy Office of Science. The SDSS-III web site is http://www.sdss3.org/.

SDSS-III is managed by the Astrophysical Research Consortium for the Participating Institutions of the SDSSIII Collaboration including the University of Arizona, the Brazilian Participation Group, Brookhaven National Laboratory, Carnegie Mellon University, University of Florida, the French Participation Group, the German Participation Group, Harvard University, the Instituto de Astrofisica de Canarias, the Michigan State/Notre Dame/JINA Participation Group, Johns Hopkins University, Lawrence Berkeley National Laboratory, Max Planck Institute for Astrophysics, Max Planck Institute for Extraterrestrial Physics, New Mexico State University, New York University, Ohio State University, Pennsylvania State University, University of Portsmouth, Princeton University, the Spanish Partic- ipation Group, University of Tokyo, University of Utah, Vanderbilt University, University of Virginia, University of Washington, and Yale University.

\section{REFERENCES}

Ahn, C. P., et al., 2014, ApJS, 211, 17

Aguerri, J. A. L., \& Sánchez-Janssen, R., 2010, A\&A, 521, A28

Aihara, H., et al., 2011, ApJS, 193, 29

Athanassoula, E. 2010, Galaxies in Isolation: Exploring Nature Versus Nurture, 421, 157

Bahé, Y. M., McCarthy, I. G., Balogh, M. L., Font, A. S., 2013, MNRAS, 430, 3017

Balogh, M. L., Navarro, J. F., Morris, S. L., 2000, ApJ, 540, 113

Barrena, R., Girardi, M., Boschin, W., \& Dasí, M. 2009, A\&A, 503, 357

Barrena, R., Girardi, M., Boschin, W., De Grandi, S., Rossetti, M., 2014, MNRAS, 442, 2216

Beers, T. C., Flynn, K., \& Gebhardt, K. 1990, ApJ, 100, 32

Bekki, K., Owers, M. S., Couch, W. J., 2010, ApJL. 718, L27

Blanton, M. R., Eisenstein, D., Hogg, D. W., Schlegel, D. J., \& Brinkmann, J. 2005, ApJ, 629, 143

Boschin, W., Barrena, R., Girardi, M., 2009, A\&A, 495, 15

Boselli, A., \& Gavazzi, G. 2006, PASP, 118, 517

Bower, R. G., Terlevich, A., Kodama, T., \& Caldwell, N. 1999, Star Formation in Early Type Galaxies, 163, 211

Carlberg, R. G., Yee, H. K. C., Ellingson, E., Abraham, R., Gravel, P., Morris, S., \& Pritchet, C. J., et al. 1996, ApJ, 462,32

Carlberg, R. G., Yee, H. K. C., \& Ellingson, E. 1997, ApJ, 478, 462

Carollo, C. M., et al., 2013, ApJ, 776, 71

Cid Fernandes, R.; Mateus, A.; Sodré, L.; Stasińska, G.; Gomes, J. M. 2005, MNRAS, 358, 363-378

Cohen, S. A., Hickox, R. C., Wegner, G. A., Einasto, M., \& Vennik, J. 2014, ApJ, 783, 136

Cohen, S. A., Hickox, R. C., Wegner, G. A., 2015, ApJ, 806, 85

Colless, M., \& Dunn, A. M. 1996, ApJ, 458, 435

Cora, S. A., Vega-Martínez, C. A., Hough, T., et al. 2018, MNRAS, 479, 2

Costa, A. P., Ribeiro, A. L. B., de Carvalho, R. R., 2018, MNRASL, 473, L31

Crone Odekon, M., Hallenbeck, G., Haynes, M. P., et al. 2018, ApJ, 852, 142

de Carvalho, R. R., Ribeiro, A. L. B., Stalder, D. H., Rosa, R. R., Costa, A. P., Moura, T. C., 2017, ApJ, 154, 96

De Lucia, G., Weinmann, S., Poggianti, B. M., Arag̃̃şnSalamanca, A., Zaritsky, D., 2012, MNRAS, 423, 1277

Deshev, B., Finoguenov, A., Verdugo, M., et al., 2017, A\&A, 607, A131

Dressler, A. 1980, ApJ, 236, 351

Dressler, A., \& Shectman, S. A. 1988, Astronomical Journal , 95, 985

Ebeling, H., Stephenson, L. N., \& Edge, A. C. 2014, ApJ, 781, L40

Einasto, M., Vennik, J., Nurmi, P., et al., 2012, A\&A, 540, A123

Ferrari, C., Benoist, C., Maurogordato, S., Cappi, A., \& Slezak, E. 2005, A\&A, 430, 19

Ferrari, C., Arnaud, M., Ettori, S., Maurogordato, S., \& Rho, J. 2006, A\&A, 446, 417

Fontanot, F., De Lucia, G., Monaco, P., Somerville, R. S., \& Santini, P. 2009, MNRAS, 397, 1776

Fujita, Y., Takizawa, M., Nagashima, M., Enoki, M., 1999, PASJ, $51, \mathrm{~L} 1$

Fujita, Y., \& Nagashima, M. 1999, ApJ, 516, 619

Gao, L., White, S. D. M., Jenkins, A., Stoehr, F., Springel, V., 2004, MNRAS, 355, 819 
Guennou, L., et al., 2014, A\&A, 561, A112

Girardi, M., Giuricin, G., Mardirossian, F., Mezzetti, M., Boschin, W., 1998, ApJ, 505, 74-95

Hernández-Fernández, J. D., Vílchez, J. M., Iglesias-Páramo, J., 2012, ApJ, 751, 54

Hogg, D. W., Blanton, M. R., Eisenstein, D. J., et al. 2003, ApJ, 585, L5

Hou, A., Parker, L. C., Wilman, D. J., et al. 2012, MNRAS, 421, 3594

Hou, A., Parker, L. C., \& Harris, W. E. 2014, MNRAS, 442, 406

Hwang, H. S., \& Lee, M. G. 2009, MNRAS, 397, 2111

Hwang, J.-S., Park, C., Banerjee, A., \& Hwang, H. S. 2018, ApJ, 856,160

Jaffé, Y. L., Verheijen, M. A. W., Haines, C. P., et al. 2016, MNRAS, 461, 1202

Jõeveer, M., Einasto, J., \& Tago, E. 1978, MNRAS, 185, 357

Kapferer, W., Ferrari, C., Domainko, W., et al. 2006, A\&A, 447, 827

Kauffmann, G., Colberg, J. M., Diaferio, A., \& White, S. D. M. 1999, MNRAS, 303, 188

Kauffmann, G., White, S. D. M., Heckman, T. M., et al. 2004, MNRAS, 353, 713

Kelkar, K., Gray, M. E., Aragón-Salamanca, A., et al. 2017, MNRAS, 469, 4551

King, I. 1962, AJ, 67, 471

Lopes, P. A. A., de Carvalho, R. R., Kohl-Moreira, J. L., \& Jones, C. 2009, MNRAS, 392, 135

Lopes, P. A. A., Ribeiro, A. L. B., \& Rembold, S. B. 2014, MNRAS, 437, 2430

Ludlow, A. D., Navarro, J. F., Springel, V., et al. 2009, ApJ, 692, 931

Ma, C.-J., Ebeling, H., Marshall, P., Schrabback, T., 2010, MNRAS, 406, 121

Mansheim, A. S., Lemaux, B. C., Dawson, W. A., Lubin, L. M., Wittman, D., Schmidt, S., 2017, ApJ, 834, 205

Mateus, A.; Sodré, L.; Cid Fernandes, R.; Stasińska, G.; Schoenell, W.; Gomes, J. M. 2006, MNRAS, 370, 721-737

Merritt D., 1988, The Minnesota lectures on Clusters of Galaxies and Large-Scale Structure

Natarajan, P., Volker, S., 2004, ApJ, 617, L13

Natarajan, P., et al., 2017, MNRAS, 468, 1962

Neistein, E., van den Bosch, F. C., \& Dekel, A. 2006, MNRAS, 372,933

Oh, S., Kim, K., Lee, J. H., et al. 2018, ApJS, 237, 14

Olave-Rojas, D., Cerulo, P., Demarco, R., et al. 2018, MNRAS, 479, 2328

Parekh, V., van der Heyden, K., Ferrari, C., Angus, G., Holwerda, B. 2006, A\&A, 575, A127

Park, C., Hwang, H. S., 2009, ApJ, 699, 1595

Peng, Y.-j., Lilly, S. J., Kovač, K., et al. 2010, ApJ, 721, 193

Peng, Y., Maiolino, R., Cochrane, R., 2015, Nature, 521, 192

Pranger, F., et al., 2013, A\&A, 557, A62

Pranger, F., et al., 2014, A\&A, 570, A40

Press, W. H., \& Schechter, P. 1974, ApJ, 187, 425

Poudel, A., Heinämäki, P., Tempel, E., Einasto, M., Lietzen, H., Nurmi, P., 2017, A\&A, 597, A86

C. Fraley and A. E. Raftery. MCLUST: Software for model-based cluster analysis. Journal of Classification, 16:297âĂŞ306, 1999.

Ribeiro, A. L. B., Lopes, P. A. A., \& Trevisan, M. 2010, MNRAS, 409, L124

Ribeiro, A. L. B., Lopes, P. A. A., Rembold, S. B., 2013, A\&A, 556, A74

Ribeiro, A. L. B., de Carvalho, R. R., Trevisan, M., Capelato, H. V., La Barbera, F., Lopes, P. A. A., Schilling, A. C., 2013, MNRAS, 434, 784

Roberts, I. D., Parker, L. C., Joshi, G. D., \& Evans, F. A., 2015, MNRAS, 448, L1

Roberts, I. D., Parker, L. C., 2017, MNRAS, 467, 3268
Roediger, E., Brüggen, M., Owers, M. S., Ebeling, H., \& Sun, M. 2014, MNRAS, 443, L114

Ruggiero, R., \& Lima Neto, G. B. 2017, MNRAS, 468, 4107

Sánchez-Blázquez, P., Peletier, R. F., Jiménez-Vicente, J., et al. 2006, MNRAS, 371, 703

Schwinn, J., Jauzac, M., Baugh, C. M., Bartelmann, M., Eckert, D., Harvey, D., Natarajan, P., Massey, R., 2017, MNRAS, 467, 2913

Shi, J., Lapi, A., Mancuso, C., Wang, H., Danese, L., 2017, ApJ, 843,104

Solanes, J. M., Salvador-Solé, E., \& González-Casado, G., 1999, A\&A, 343, 733

Stroe, A., Oosterloo, T., Röttgering, H. J. A., Sobral, D., van Weeren, R., Dawson, W., 2015, MNRAS, 452, 2731

Sybilska, A., Lisker, T., Kuntschner, H., et al. 2017, MNRAS, 470,815

Taranu, D. S., Hudson, M. J., Balogh, M. L., Smith, R. J., Power, C., Oman, K. A., Krane, B., 2014, MNRAS, 440, 1934

Tempel, E., Tago, E., \& Liivamägi, L. J., 2012, A\&A, 540, A106

van den Bergh, S. 1999, The Publications of the Astronomical Society of the Pacific, 111, 657

van den Bosch, F. C., Jiang, F., 2016, MNRAS, 458, 2870

Vijayaraghavan, R., Ricker, P. M., 2013, MNRAS, 435, 2713

Wetzel, A. R., Tinker, J. L., Conroy, C., \& van den Bosch, F. C. 2013, MNRAS, 432, 336

Wegner, G. A., Chu, D. S., Hwang, H. S., 2015, MNRAS, 447, 1126

Wu, P.-F., Gal, R. R., Lemaux, B. C., et al. 2014, ApJ, 792, 16

Yan, P.-F., Li, F., \& Yuan, Q.-R., 2015, Research in Astronomy and Astrophysics, 15, 1773

Zhu, G., Blanton, M. R., \& Moustakas, J. 2010, ApJ, 722, 491

This paper has been typeset from a TEX/LATEX file prepared by the author. 